\title{
NECESSARY AND SUFFICIENT CONDITIONS FOR THE NONINCREASE OF SCALAR FUNCTIONS ALONG SOLUTIONS TO CONSTRAINED DIFFERENTIAL INCLUSIONS*
}

\author{
Mohamed Maghenem ${ }^{1, * *}$, Alessandro Melis $^{2}$ \\ AND RicARDO G. SANFELICE ${ }^{3}$
}

\begin{abstract}
In this paper, we propose necessary and sufficient conditions for a scalar function to be nonincreasing along solutions to general differential inclusions with state constraints. The problem of determining if a function is nonincreasing appears in the study of stability and safety, typically using Lyapunov and barrier functions, respectively. The results in this paper present infinitesimal conditions that do not require any knowledge about the solutions to the system. Results under different regularity properties of the considered scalar function are provided. This includes when the scalar function is lower semicontinuous, locally Lipschitz and regular, or continuously differentiable.
\end{abstract}

Mathematics Subject Classification. 93A10-26B05.

Received May 8, 2021. Accepted January 26, 2022.

\section{INTRODUCTION}

\subsection{Background}

The problem considered in this paper is to characterize, via necessary and sufficient conditions, the property of a function to be nonincreasing when evaluated along the solutions to a nonlinear system. In the particular case where the system is given by $\dot{x}=F(x)$ and the function is $B: \mathbb{R}^{n} \rightarrow \mathbb{R}$, this problem consists in establishing necessary and sufficient conditions such that the scalar function $t \mapsto B(\phi(t))$ is nonincreasing for every solution $t \mapsto \phi(t)$ to $\dot{x}=F(x)$. For such conditions to be useful, they need to be infinitesimal, meaning that they do not depend on the solutions; namely, they only involve $B$ and $F$. The aforementioned problem is known to be one of the fundamental problems in calculus [7], and has attracted the attention of mathematicians over the years, dating back to the work of Pierre de Fermat on local extrema for differentiable functions in the $17^{\text {th }}$ century [4].

* Research partially supported by NSF Grants no. ECS-1710621, CNS-1544396, and CNS-2039054, by AFOSR Grants no. FA9550-19-1-0053, FA9550-19-1-0169, and FA9550-20-1-0238, and by CITRIS and the Banatao Institute at the University of California.

Keywords and phrases: Constrained systems; Differential inclusions; Nonincreasing functions; Lyapunov-like functions.

${ }^{1}$ University Grenoble Alpes, CNRS, Grenoble INP, Gipsa lab, Grenoble, France.

2 Department of Electrical, Electronic, and Information Engineering, University of Bologna, Italy.

3 Department of Electrical \& Computer Engineering, University of California Santa Cruz, CA, USA.

** Corresponding author: mohamed.maghenem@gipsa-lab.fr 
A key difficulty in solving such a problem emerges from the smoothness of (or lack of) the maps $F$ and $B$. As expected, initial solutions to this problem deal with the particular case where both $F$ and $B$ are sufficiently smooth. In such a basic setting, a necessary and sufficient condition for $B$ to be nonincreasing is that the scalar product between the gradient of $B$ and $F$ is nonpositive at each $x \in \mathbb{R}^{n}$; namely, $\langle\nabla B(x), F(x)\rangle \leq 0$ for all $x \in \mathbb{R}^{n}$. When $B$ is not continuously differentiable, the problem requires nonsmooth analysis tools since the gradient of $B$ may not be defined according to the classical sense.

When $B$ is nonsmooth, the existing solutions to the problem use the notion of directional subderivatives $[11,28,29]$. It appears that directional subderivatives were first proposed and used by Ulisse Dini in 1878 [15]. Since then, many extensions were proposed in the literature, see [3, 12-14]. These extensions allow to cover general scenarios where $F$ is a general set-valued map, and thus the system is a differential inclusion of the form $\dot{x} \in F(x)$, and $B$ is merely continuous, or just semicontinuous. Moreover, in those extensions, the classical gradient $\nabla B$ is replaced by its nonsmooth versions, such as the proximal subderivative [8], denoted $\partial_{P} B$, and the Clarke generalized gradient $[5,9]$, denoted $\partial_{C} B$.

\subsection{Motivation}

To the best of our knowledge, the existing solutions to the stated problem consider a system $\dot{x} \in F(x)$ defined on an open subset $C \subset \mathbb{R}^{n}$ where the solutions cannot start from the boundary of the set $C$, denoted $\partial C$. This requirement is customarily used in the literature of unconstrained systems, see, e.g., [3, 13, 18]. However, the assumption that the solutions cannot start from $\partial C$ is restrictive when dealing with general constrained systems of the form

$$
\mathcal{H}_{f}: \quad \dot{x} \in F(x) \quad x \in C \subset \mathbb{R}^{n},
$$

where $C$ is not necessarily open and the solutions might start from or slide on $\partial C$.

In this context of constrained systems, the existing solutions to the considered problem are not applicable. Indeed, assume that the set $C$ is closed. In this case, it might be possible to find a vector $\eta \in F(x)$ for some $x \in \partial C$ such that the direction $\eta$ does not generate solutions, for example, when $\eta$ points towards the complement of $C$. Such vectors should not be included in an infinitesimal condition for the nonincrease of $t \mapsto B(\phi(t))$, otherwise this condition would not be necessary; see the forthcoming Example 4.2 for more details. At the same time, the vector $\eta$, although not generating solutions, may affect the global behavior of the solutions. Hence, such vectors should be somehow included in the characterization of the nonincrease of $t \mapsto B(\phi(t))$, otherwise the condition may fail to be sufficient; see the forthcoming Example 4.3 for more details. As we show in this paper, to handle such a compromise, extra assumptions relating $F$ to the boundary of $C$ must be imposed.

Solving the considered problem in the context of constrained systems finds a natural motivation when characterizing safety in terms of barrier functions. Indeed, characterizing the nonincreasing behavior of such functions along solutions is critical for the safety property to hold.

\subsection{Contributions}

In this paper, we propose solutions to the stated problem in the general case of constrained differential inclusions. This problem is studied under different conditions on the scalar function $B$, including the following three cases:

- When the scalar function $B$ is lower semicontinuous (i.e., for each $x \in \mathbb{R}^{n}$ and for each sequence $\left\{x_{n}\right\}_{n=0}^{\infty} \subset \mathbb{R}^{n}$ with $\liminf _{n \rightarrow \infty} x_{n}=x \in \mathbb{R}^{n}$, we have $\left.\liminf _{n \rightarrow \infty} B\left(x_{n}\right) \geq B(x)\right)$ we transform the problem of showing that $B$ is nonincreasing along the solutions to $\mathcal{H}_{f}=(C, F)$ into characterizing forward pre-invariance of the set epi $B \cap(\operatorname{cl}(C) \times \mathbb{R})$, where epi $B:=\left\{(x, r) \in \mathbb{R}^{n} \times \mathbb{R}: r \geq B(x)\right\}$ is the epigraph 
of $B$ and $\operatorname{cl}(C)$ is the closure of $C$, for the augmented constrained system

$$
\left[\begin{array}{c}
\dot{x} \\
\dot{r}
\end{array}\right] \in\left[\begin{array}{c}
F(x) \\
0
\end{array}\right] \quad(x, r) \in C \times \mathbb{R}
$$

Namely, we propose necessary and sufficient conditions guaranteeing that each solution to (1.2) starting from epi $B \cap(\operatorname{cl}(C) \times \mathbb{R})$ never leaves this set for all time instants at which it is defined. As a consequence, the proposed conditions are inequalities involving $F$, the proximal subdifferential of $B$, denoted $\partial_{P} B$, and the contingent cone to $C$, denoted $T_{C}$, which is, roughly speaking, used to rule out directions of $F$ not generating solutions.

- When the function $B$ is locally Lipschitz, similar inequalities to the lower semicontinuous case are proposed. Due to the assumption of a stronger smoothness property for $B$, the Clarke generalized gradient, denoted $\partial_{C} B$, is used instead of the proximal subdifferential $\partial_{P} B$.

- When the function $B$ is continuously differentiable, the conditions proposed are a corollary of those when $B$ is locally Lipschitz. In particular, when $B$ is continuously differentiable the Clarke generalized gradient $\partial_{C} B$ reduces to the classical gradient $\nabla B$.

To the best of our knowledge, there are no results in the literature characterizing the nonincrease of $t \mapsto$ $B(\phi(t))$, for each $\phi$ solution to $\mathcal{H}_{f}=(C, F)$, using necessary and sufficient infinitesimal conditions. A preliminary version of this paper is in the conference article [19], where the proofs, detailed explanations, and some examples have been omitted.

\subsection{Notations and preliminaries}

For $x, y \in \mathbb{R}^{n}, x^{\top}$ denotes the transpose of $x,|x|$ the Euclidean norm of $x,\langle x, y\rangle:=x^{\top} y$ the scalar product between $x$ and $y$, and co $\{x, y\}$ the set of all convex combinations between $x$ and $y$. For a scalar function $B: \mathbb{R}^{n} \rightarrow \mathbb{R}, \nabla B(x)$ denotes the gradient of the function $B$ evaluated at $x$. Note that the epigraph of a lower semicontinuous function $B$ is a closed subset of $\mathbb{R}^{n+1}$. By $\mathbb{B}$ we denote the closed unit ball in $\mathbb{R}^{n}$ centered at the origin. For a subset $K \subset \mathbb{R}^{n}$, we use $|x|_{K}:=\inf _{y \in K}|x-y|$ to denote the distance from $x$ to $K$, int $(K)$ to denote the interior of $K, \partial K$ its boundary, and $U(K)$ to denote a sufficiently small open neighborhood around $K$. For $O \subset \mathbb{R}^{n}$, we use $K \backslash O$ to denote the subset of elements of $K$ that are not in $O$. Furthermore, we use $T_{K}(x), C_{K}(x), N_{K}(x)$, and $N_{K}^{P}(x)$ to denote, respectively, the contingent, the Clarke tangent, the normal ${ }^{1}$, and the proximal normal cones of $K$ at $x$ given by $T_{K}(x):=\left\{v \in \mathbb{R}^{n}: \liminf _{h \rightarrow 0^{+}}|x+h v|_{K} / h=0\right\}, C_{K}(x):=\left\{v \in \mathbb{R}^{n}: \lim _{y \rightarrow x, h \rightarrow 0^{+}}|y+h v|_{K} / h=0\right\}$, $N_{K}(x):=\left\{v \in \mathbb{R}^{n}:\langle v, w\rangle \leq 0 \quad \forall w \in T_{K}(x)\right\}$, and $N_{S}^{P}(x):=\left\{\zeta \in \mathbb{R}^{m}: \exists r>0:|x+r \zeta|_{S}=r|\zeta|\right\}$. Finally, for a set-valued map $F: \mathbb{R}^{m} \rightrightarrows \mathbb{R}^{n}$,

- $F$ is outer semicontinuous at $x \in \mathbb{R}^{m}$ if, for all $\left\{x_{i}\right\}_{i=0}^{\infty} \subset \mathbb{R}^{m}$ and for all $\left\{y_{i}\right\}_{i=0}^{\infty} \subset \mathbb{R}^{n}$ with $x_{i} \rightarrow x$, $y_{i} \in F\left(x_{i}\right)$, and $y_{i} \rightarrow y \in \mathbb{R}^{n}$, we have $y \in F(x)$; see Definition 5.9 of [17].

- $F$ is lower semicontinuous (or, equivalently, inner semicontinuous) at $x \in \mathbb{R}^{m}$ if, for each $\epsilon>0$ and for each $y_{x} \in F(x)$, there exists $U(x)$ a neighborhood of $x$ such that, for each $z \in U(x)$, there exists $y_{z} \in F(z)$ such that $\left|y_{z}-y_{x}\right| \leq \epsilon$; see Proposition 2.1 of [23].

- $F$ is upper semicontinuous at $x \in \mathbb{R}^{m}$ if, for each $\epsilon>0$, there exists $U(x)$ such that, for each $y \in U(x)$, $F(y) \subset F(x)+\epsilon \mathbb{B}$; see Definition 1.4.1 of [4].

- $F$ is continuous at $x \in \mathbb{R}^{m}$ if it is both upper and lower semicontinuous at $x$.

- $F$ is outer (lower, and upper, respectively) semicontinuous if it is outer (lower, and upper, respectively) semicontinuous at every $x \in \mathbb{R}^{m}$. Finally, $F$ is said to be continuous if it is continuous at every $x \in \mathbb{R}^{m}$.

- $F$ is locally bounded if, for each $x \in \mathbb{R}^{n}$, there exist $U(x)$ and $K>0$ such that $|\zeta| \leq K$ for all $\zeta \in F(y)$, and for all $y \in U(x)$.

\footnotetext{
${ }^{1}$ Also named subnormal cone in [2].
} 
- $F$ is locally Lipschitz if, for each compact set $K \subset \mathbb{R}^{n}$, there exists $k>0$ such that, for each $x \in K$ and $y \in K, F(y) \subset F(x)+k|x-y| \mathbb{B}$.

\section{Constrained Differential inClusions}

Consider the constrained differential inclusion $\mathcal{H}_{f}:=(C, F)$ in (1.1) with the state variable $x \in \mathbb{R}^{n}$, the set $C \subset \mathbb{R}^{n}$ and the set-valued map $F: \mathbb{R}^{n} \rightrightarrows \mathbb{R}^{n}$. As opposed to the existing literature dealing with unconstrained differential inclusions, where $C=\mathbb{R}^{n}[3,13]$, the set $C$ in (1.1) is not necessarily open and does not neccessarily correspond to $\mathbb{R}^{n}$. Next, we introduce the concept of a solution to $\mathcal{H}_{f}$.

Definition 2.1. (Concept of Solution to $\mathcal{H}_{f}$ ) A function $\phi: \operatorname{dom} \phi \rightarrow \mathbb{R}^{n}$ with $\operatorname{dom} \phi \subset \mathbb{R}_{\geq 0}$ and $t \mapsto \phi(t)$ locally absolutely continuous is a solution to $\mathcal{H}_{f}$ if

(S1) $\phi(0) \in \mathrm{cl}(C)$

(S2) $\phi(t) \in C \quad$ for all $t \in \operatorname{int}(\operatorname{dom} \phi)$,

(S3) $\frac{d \phi}{d t}(t) \in F(\phi(t)) \quad$ for almost all $t \in \operatorname{dom} \phi$.

Remark 2.2. Condition (S1) allows solutions starting from $\partial C \backslash C$ to flow into $C$ such that (S2) is satisfied. Furthermore, (S2) allows solutions starting from $C$ to reach $\partial C \backslash C$. Hence, symmetry between forward and backward solutions is preserved.

A solution $\phi$ to $\mathcal{H}_{f}$ is said to be maximal if there is no solution $\psi$ to $\mathcal{H}_{f}$ such that $\phi(t)=\psi(t)$ for all $t \in \operatorname{dom} \phi$ with $\operatorname{dom} \phi$ a proper subset of $\operatorname{dom} \psi$. Furthermore, it is said to be forward complete if dom $\phi$ is unbounded. Finally, we recall the definitions of forward pre-invariance and pre-contractivity of a set $K \subset \mathbb{R}^{n}$ for the system $\mathcal{H}_{f}$.

Definition 2.3 (Forward pre-Invariance). A set $K \subset \mathbb{R}^{n}$ is said to be forward pre-invariant for a constrained system $\mathcal{H}_{f}=(C, F)$ if each solution to $\mathcal{H}_{f}$ starting from $K$ remains in it.

Definition 2.4 (Pre-contractivity). A closed set $K \subset \mathbb{R}^{n}$ is said to be pre-contractive for a constrained system $\mathcal{H}_{f}=(C, F)$ if, for every nontrivial solution, i.e., solution whose domain contains more than one element, $\phi$ starting from $x_{o} \in \partial K$, there exists $\epsilon>0$ such that $\phi(t) \subset \operatorname{int}(K)$ for all $t \in(0, \epsilon]$.

The "pre" in forward pre-invariance and forward pre-contractivity is used to accommodate maximal solutions that are not complete.

Throughout this paper the set-valued map $F$ satisfies the following mild assumption.

Assumption 2.5. $F: \mathbb{R}^{n} \rightrightarrows \mathbb{R}^{n}$ is upper semicontinuous and $F(x)$ is compact and convex for all $x \in \mathbb{R}^{n}$.

Before concluding this section, the following remarks are in order.

Remark 2.6. Assumption 2.5 is customarily used in the literature as the tightest requirement for the existence of solutions and adequate structural properties for the set of solutions, see $[2,3,13]$. When $F$ is single valued, Assumption 2.5 reduces to the continuity of $F$. In some of the existing literature, e.g. [17], Assumption 2.5 is replaced by the equivalent assumption stating that $F$ needs to be outer semicontinuous and locally bounded with convex images. Indeed, outer semicontinuous and locally bounded set-valued maps are upper semicontinuous with compact images ([25], Thm. 5.19), the converse is also true using Lemma 5.15 of [17] and the fact that upper semicontinuous set-valued maps with compact images are locally bounded.

Remark 2.7. Constrained differential inclusions $\mathcal{H}_{f}=(C, F)$ constitute a key component in the modeling of hybrid systems. Indeed, according to [17], a general hybrid system modeled as a hybrid inclusion is given by

$$
\mathcal{H}:\left\{\begin{array}{cc}
\dot{x} \in F(x) & x \in C \\
x^{+} \in G(x) & x \in D
\end{array}\right.
$$


where, in addition to the continuous dynamics or flows $\mathcal{H}_{f}=(C, F)$, the discrete dynamics are defined by the jump set $D \subset \mathbb{R}^{n}$ and the jump map $G: \mathbb{R}^{n} \rightrightarrows \mathbb{R}^{n}$. Furthermore, solutions to $\mathcal{H}_{f}=(C, F)$ correspond to solutions to $\mathcal{H}$, according to Definition 2.6 of [17], that never jump.

\section{Problem statement, motivational application, and existing SOLUTIONS}

In this section, we formulate the problem treated in this paper. After that, we illustrate a motivation from stability and safety analysis using Lyapunov and barrier functions, respectively.

Given a constrained differential inclusion $\mathcal{H}_{f}=(C, F)$ as in (1.1) and a scalar function $B: \mathbb{R}^{n} \rightarrow \mathbb{R}$, we would to address the following problem.

Problem 3.1. Provide necessary and sufficient infinitesimal conditions (involving only $B, F$, and the set $C$ ) such that the following property holds:

( T) The scalar function $B$ is nonincreasing along the solutions to $\mathcal{H}_{f}$; namely, for every solution $t \mapsto \phi(t)$ to $\mathcal{H}_{f}$, the map $t \mapsto B(\phi(t))$ is nonincreasing ${ }^{2}$.

\subsection{Motivational application}

In addition to the theoretical motivation mentioned in Section 1.2, Problem 3.1 naturally emerges when studying safety for hybrid systems using barrier functions [21, 22]. More precisely, given a hybrid system of the form $\mathcal{H}:=(C, F, D, G)$ (see Rem. 2.7), given a set of initial conditions $X_{o} \subset \operatorname{cl}(C) \cup D$ and an unsafe set $X_{u} \subset \mathbb{R}^{n}$, the hybrid system $\mathcal{H}$ is said to be safe with respect to $\left(X_{o}, X_{u}\right)$ if the solutions starting from $X_{o}$ never reach the set $X_{u}$. To certify safety with respect to $\left(X_{o}, X_{u}\right)$, scalar functions $B: \mathbb{R}^{n} \rightarrow \mathbb{R}$ satisfying

$$
\begin{aligned}
& B(x)>0 \quad \forall x \in X_{u} \\
& B(x) \leq 0 \quad \forall x \in X_{o}
\end{aligned}
$$

named barrier function candidates, is used in [1, 16, 24], among many others. A barrier function candidate guarantees safety for $\mathcal{H}$ with respect to $\left(X_{o}, X_{u}\right)$ if the following properties hold:

$$
B(\eta) \leq 0 \quad \forall \eta \in G(x), \quad \forall x \in K \cap D, \quad \text { and }
$$

( $\star$ ) The function $B$ is nonincreasing along the solutions to $\mathcal{H}_{f}=(C \backslash \operatorname{int}(K), F)$, where

$$
K:=\{x \in \operatorname{cl}(C) \cup D: B(x) \leq 0\} .
$$

In particular, $(\star \star)$ guarantees that solutions to $\mathcal{H}$ from $K$ cannot flow out of $K$, while (3.3) assures that such solutions cannot jump from $K \cap D$ to a point outside of $K$. Note that condition (3.3) is already infinitesimal. Furthermore, we recover in $(\star \star)$ the non-increase condition along the solutions to a constrained system. Hence, it is natural that one wants to replace $(\star \star)$ by sufficient infinitesimal conditions, which will depend on whether $B$ is smooth or not.

On the other hand, the converse safety problem pertains to showing, when $\mathcal{H}$ is safe with respect to $\left(X_{o}, X_{u}\right)$, the existence of a barrier function candidate $B: \mathbb{R}^{n} \rightarrow \mathbb{R}$ such that (3.3) and ( $\left.\star \star\right)$ are satisfied. Note that this converse problem is addressed in [21,22] by constructing a barrier function $B$ that depends on both $x$ and the (hybrid) time. However, one still needed to show that the constructed barrier function enjoys some smoothness properties to replace $(\star \star)$ by an equivalent infinitesimal condition - which, as pointed out in Section 1.1, is a solution-independent condition (as in Lyapunov stability theory). The latter is addressed for unconstrained continuous-time systems in [20]. However, once tackling the constrained case, Problem 3.1 is faced.

\footnotetext{
${ }^{2}$ Or, equivalently, $B\left(\phi\left(t_{1}\right)\right) \leq B\left(\phi\left(t_{2}\right)\right)$ for all $\left(t_{1}, t_{2}\right) \in \operatorname{dom} \phi \times \operatorname{dom} \phi$ with $t_{1} \geq t_{2}$.
} 


\subsection{Existing results in the unconstrained case}

Existing solutions to Problem 3.1 in the unconstrained case, i.e. $C=\mathbb{R}^{n}$, include the ones listed below ${ }^{3}$ :

- When $n=1, B$ is continuously differentiable, and $F \equiv 1$ : the function $B$ is nonincreasing along the solutions to $\mathcal{H}_{f}=(C, F)$ if and only if $\nabla B(x) \leq 0$ for all $x \in \mathbb{R}$.

- When $n \geq 1$, and $F$ satisfies Assumption 2.5, the continuously differentiable function $B$ is nonincreasing along the solutions to $\mathcal{H}_{f}=(C, F)$ if

$$
\langle\nabla B(x), \zeta\rangle \leq 0 \quad \forall \zeta \in F(x), \quad \forall x \in \mathbb{R}^{n} .
$$

The equivalence is true when, additionally, $F$ is continuous.

- When the function $B$ is only continuous, the standard gradient $\nabla B$ cannot be used. Existing solutions to Problem 3.1, in this case, use the directional subderivative. Indeed, in the simple case where $n=1$ and $F \equiv 1$, the following result is available in page 3 of [13].

Lemma 3.2. A continuous function $B: \mathbb{R} \rightarrow \mathbb{R}$ is nonincreasing if and only if

$$
D B(x):=\liminf _{t \rightarrow 0^{+}} \frac{B(x+t)-B(x)}{t} \leq 0 \quad \forall x \in \mathbb{R} .
$$

- When $n \geq 1, F$ satisfies Assumption 2.5, B is nonincreasing along the solutions to $\mathcal{H}_{f}=\left(\mathbb{R}^{n}, F\right)$ if

$$
\liminf _{\substack{w \rightarrow \zeta \\ t \rightarrow 0^{+}}} \frac{B(x+t w)-B(x)}{t} \leq 0 \quad \forall \zeta \in F(x), \quad \forall x \in \mathbb{R}^{n}
$$

The equivalence is true when, additionally, $F$ is continuous; see [3, 28].

- When $n \geq 1, F$ satisfies Assumption 2.5, and $B$ is locally Lipschitz, $B$ is nonincreasing along the solutions to $\mathcal{H}_{f}=\left(\mathbb{R}^{n}, F\right)$ if $[9,27]$

$$
\langle\eta, \zeta\rangle \leq 0 \quad \forall \zeta \in F(x), \quad \forall \eta \in \partial_{C} B(x), \quad \forall x \in \mathbb{R}^{n} .
$$

The equivalence is true when, additionally, $F$ is continuous and $B$ is regular. Recall that $\partial_{C} B: \mathbb{R}^{n} \rightrightarrows \mathbb{R}^{n}$ is the Clarke generalized gradient of $B$, which, according to the equivalence in Theorem 8.1, page 93 of [13], can be defined as follows.

Definition 3.3 (Clarke generalized gradient). Let $\Omega$ be any subset of zero measure in $\mathbb{R}^{n}$, and let $\Omega_{B}$ be the zero-measure set of points in $\mathbb{R}^{n}$ at which $B$ fails to be differentiable. Then, the Clarke generalized gradient at $x$ is defined as

$$
\partial_{C} B(x):=\operatorname{co}\left\{\lim _{i \rightarrow \infty} \nabla B\left(x_{i}\right): x_{i} \rightarrow x, x_{i} \notin \Omega_{B}, x_{i} \in \Omega\right\} .
$$

Furthermore, the regularity of $B$ is defined below, following Proposition 7.3, page 91 of [13].

Definition 3.4 (Regular functions). A locally Lipschitz function $B: \mathbb{R}^{n} \rightarrow \mathbb{R}$ is regular if epi $B$ is regular; namely, $T_{\mathrm{epi} B}(x)=C_{\mathrm{epi} B}(x)$ for all $x \in \operatorname{epi} B$.

\footnotetext{
${ }^{3}$ The first two solutions can be derived easily.
} 
- When $n \geq 1, F$ satisfies Assumption 2.5, and $B$ is locally Lipschitz and regular, $B$ is nonincreasing along the solutions to $\mathcal{H}_{f}=\left(\mathbb{R}^{n}, F\right)$ if $[5,6,18]$

$$
\langle\eta, \zeta\rangle \leq 0 \quad \forall \eta \in \partial_{C} B(x), \quad \forall \zeta \in F(x): \exists c \in \mathbb{R}:\langle\eta, \zeta\rangle=c \text { for all } \eta \in \partial_{C} B(x), \quad \forall x \in \mathbb{R}^{n} .
$$

Equivalence holds when, additionally, $F$ is continuous. Compared to (3.7), in (3.9), we check the inequality only for vector fields that yield the same scalar product with all the vectors in $\partial_{C} B$.

- When $n \geq 1, F$ satisfies Assumption 2.5 and, additionally, $F$ is continuous, and $B$ is locally Lipschitz, $B$ is nonincreasing along the solutions to $\mathcal{H}_{f}=\left(\mathbb{R}^{n}, F\right)$ if and only if [26]

$$
\langle\nabla B(x), \zeta\rangle \leq 0 \quad \forall \zeta \in F(x), \quad \forall x \in \mathbb{R}^{n} \text { such that } \nabla B(x) \text { exists. }
$$

Compared to (3.7), in (3.10), we check the inequality for all vectors in $F(x)$ but at points $x$ where the gradient of $B$ is well defined.

- When the function $B$ is lower semicontinuous, $n \geq 1$, and $F$ is locally Lipschitz with closed and convex images, $B$ is nonincreasing along the solutions to $\mathcal{H}_{f}=\left(\mathbb{R}^{n}, F\right)$ if and only if Theorem 6.3 of [13]

$$
\langle\eta, \zeta\rangle \leq 0 \quad \forall \zeta \in F(x), \quad \forall \eta \in \partial_{P} B(x), \quad \forall x \in \mathbb{R}^{n},
$$

where $\partial_{P} B: \mathbb{R}^{n} \rightrightarrows \mathbb{R}^{n}$ is the proximal subdifferential of $B$, which is defined below.

Definition 3.5 (Proximal subdifferential [13]). The proximal subdifferential of a function $B: \mathbb{R}^{n} \rightarrow \mathbb{R}$ is the set-valued map $\partial_{P} B: \mathbb{R}^{n} \rightrightarrows \mathbb{R}^{n}$ such that, for all $x \in \mathbb{R}^{n}$,

$$
\partial_{P} B(x):=\left\{\eta \in \mathbb{R}^{n}:\left[\eta^{\top}-1\right]^{\top} \in N_{\mathrm{epi} B}^{P}(x, B(x))\right\} .
$$

Remark 3.6. When $B$ is twice continuously differentiable, $\partial_{P} B(x)=\{\nabla B(x)\}$. Moreover, the latter equality holds also when $B$ is only continuously differentiable provided that $\partial_{P} B(x) \neq \emptyset$.

\section{Challenges in the CONStRained CASE}

In this section, we illustrate why the conditions in (3.7)-(3.11) do not solve Problem 3.1 in the general constrained case. For this purpose, we introduce the following useful set $\tilde{C}$ :

$$
\tilde{C}:=\{x \in \operatorname{cl}(C): \exists \phi \in \mathcal{S}(x), \operatorname{dom} \phi \neq\{0\}\},
$$

where $\mathcal{S}(x)$ is the set of solutions starting from $x$.

Remark 4.1. For a constrained system $\mathcal{H}_{f}=(C, F)$, there are numerous solutions-independent methods to find the set $\tilde{C}$, i.e., to know whether, from $x_{o} \in C$, a nontrivial solution exists or not. In the following, we recall some of such conditions:

- When $F\left(x_{o}\right) \cap T_{C}\left(x_{o}\right)=\emptyset$, we conclude that each solution to $\mathcal{H}_{f}$ starting from $x_{o}$ is trivial; see Proposition 3.4 .1 of $[2]$.

- When there exists a neighborhood $U\left(x_{o}\right)$ such that $F(x) \cap T_{C}(x) \neq \emptyset$ for all $x \in U\left(x_{o}\right) \cap \operatorname{cl}(C)$, then there exists a non-trivial solution to $\mathcal{H}_{f}$ starting at $x_{o}$; see Proposition 3.4.2 of [2].

- When $F\left(x_{o}\right) \subset D_{C}\left(x_{o}\right)$, where

$$
D_{C}\left(x_{o}\right):=\left\{v \in \mathbb{R}^{n}: \exists \epsilon, \alpha>0: x+(0, \alpha](v+\epsilon \mathbb{B}) \subset C\right\},
$$

then there exists a nontrivial solution to $\mathcal{H}_{f}$ starting from $x_{o}$; see Theorem 4.3 .4 of [2]. 


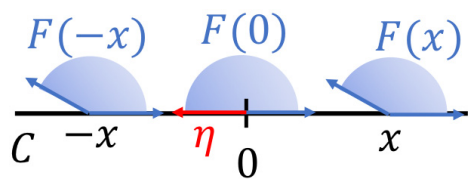

Figure 1. Illustration of the data of the system $\mathcal{H}_{f}$ in Example 4.2.

Other results can be derived when, additionally, the set $C$ is convex or $F$ is locally Lipschitz; see [13]. These techniques are well established in the literature and not within the scope of our paper. In our case, we start from a constrained system $\mathcal{H}_{f}=(C, F)$ for which we are able to find $\tilde{C}$.

When the set $\tilde{C}$ is not open; namely, nontrivial solutions to $\mathcal{H}_{f}$ start from $\partial C$, the solutions to Problem 3.1 in (3.4), (3.7), and (3.11) are not applicable. Indeed, suppose that the set $\tilde{C}$ is closed. When $x \in \partial C \cap C$, only vectors in $F(x)$ that generate nontrivial solutions should be considered in the conditions solving Problem 3.1. Otherwise, the conditions will not be necessary. In particular, the vectors in $F(x) \backslash T_{C}(x)$ must not be included. Hence, we propose to modify the conditions (3.4), (3.7), and (3.11), respectively, as:

$$
\begin{aligned}
& \langle\nabla B(x), \zeta\rangle \leq 0 \quad \forall \zeta \in F(x) \cap T_{C}(x), \quad \forall x \in \tilde{C} . \\
& \langle\eta, \zeta\rangle \leq 0 \quad \forall \zeta \in F(x) \cap T_{C}(x), \quad \forall \eta \in \partial_{C} B(x), \quad \forall x \in \tilde{C} . \\
& \langle\eta, \zeta\rangle \leq 0 \quad \forall \zeta \in F(x) \cap T_{C}(x), \quad \forall \eta \in \partial_{P} B(x), \quad \forall x \in \tilde{C} .
\end{aligned}
$$

The new conditions (4.2)-(4.4) still fail to be necessary. Indeed, in the following example, we consider a situation where $F$ is locally Lipschitz with closed and convex images, the set $C=\tilde{C}$ is closed, and the continuously differentiable function $B$ is nonincreasing along the solutions but, for some $x_{o} \in \tilde{C}$, there exist $v_{o} \in F\left(x_{o}\right) \cap$ $T_{C}\left(x_{o}\right)$ such that inequality in (4.2) is not satisfied.

Example 4.2. Consider the system $\mathcal{H}_{f}=(C, F)$ with $x \in \mathbb{R}^{2}$,

$$
F(x):=\operatorname{co}\left\{\left[\begin{array}{ll}
1 & 0
\end{array}\right]^{\top},\left[\begin{array}{ll}
-\cos \left(x_{1}^{2}\right) & \sin \left(x_{1}^{2}\right)
\end{array}\right]^{\top}\right\} \quad \forall x \in C,
$$

and $C:=\left\{x \in \mathbb{R}^{2}: x_{2}=0\right\}$ (see Fig. 1). Furthermore, consider the function $B(x):=-x_{1}$.

Note that $F$ is locally Lipschitz and has closed and convex images. Furthermore, starting from each initial condition $x_{o}:=\left[\begin{array}{ll}x_{o 1} & x_{o 2}\end{array}\right]^{\top} \in C$, the only nontrivial solution is given by $\phi(t):=\left[\begin{array}{ll}x_{o 1}+t & 0\end{array}\right]^{\top}$ for all $t \geq 0$; hence, $\tilde{C}=C$ and $B$ is nonincreasing along each nontrivial solution. However, for $x_{o}=0$, we show that, for $v_{o}:=\left[\begin{array}{ll}-1 & 0\end{array}\right]^{\top} \in F(0) \cap T_{C}(0)$, condition (4.2) is not satisfied. Indeed, we note that $\left\langle\nabla B(0), v_{o}\right\rangle=1>0$.

On the other hand, the vectors in $F(x)$ not generating solutions may affect the global behavior of the solutions in a way that they fail to render the map $t \mapsto B(\phi(t))$ nonincreasing. The latter is more likely to happen when $B$ is discontinuous. Consequently, assumptions on some elements of $F(x)$ not generating solutions should be considered, otherwise, the conditions can fail to be sufficient. In the following example, we propose a constrained system $\mathcal{H}_{f}=(C, F)$ where $F$ is locally Lipschitz with closed and convex images, the set $C$ is closed, and (4.4) is satisfied. However, the lower semicontinuous function $B$ fails to be nonincreasing along solutions.

Example 4.3. Consider the system $\mathcal{H}_{f}=(C, F)$ with $x \in \mathbb{R}^{2}$,

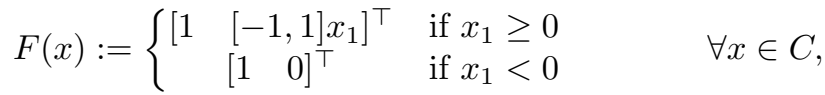

$$
\begin{aligned}
& C:=\left\{x \in \mathbb{R}^{2}:\left|x_{2}\right| \geq x_{1}^{2}\right\} \cup\left\{x \in \mathbb{R}^{2}: x_{1} \leq 0\right\} \cup\left\{x \in \mathbb{R}^{2}: x_{2}=0\right\} \text {. (See Fig. 2) }
\end{aligned}
$$




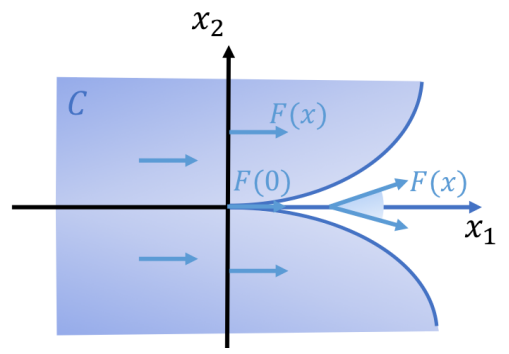

Figure 2. Illustration of the data of the system $\mathcal{H}_{f}$ in Example 4.3.

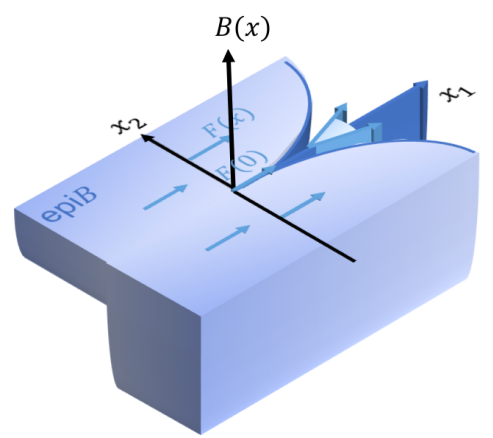

Figure 3. Illustration of the set epi $B \cap(C \times \mathbb{R})$ in Example 4.3.

Furthermore, consider the lower semicontinuous function

$$
B(x):= \begin{cases}0 & \text { if } x_{2} \leq 0 \\ 1 & \text { if } x_{2}>0\end{cases}
$$

We will show that in this case condition (4.4) holds, but the function $B$ is not nonincreasing along the solutions to $\mathcal{H}_{f}$. Indeed, we start noting that

$$
\begin{aligned}
\operatorname{epi} B & =\left\{(x, r) \in \mathbb{R}^{3}: x_{2} \leq 0, r \geq 0\right\} \cup\left\{(x, r) \in \mathbb{R}^{3}: x_{2}>0, r \geq 1\right\}, \\
\operatorname{epi} B \cap(C \times \mathbb{R}) & =\left\{(x, r) \in \mathbb{R}^{3}: x_{2} \leq 0, r \geq 0, x_{1} \leq \sqrt{-x_{2}}\right\} \cup \\
& \left\{(x, r) \in \mathbb{R}^{3}: x_{2}>0, r \geq 1, x_{1} \leq \sqrt{x_{2}}\right\} \cup\left\{(x, r) \in \mathbb{R}^{3}: x_{1} \geq 0, r \geq 0, x_{2}=0\right\} .
\end{aligned}
$$

Furthermore, note that $F$ is locally Lipschitz with closed and convex images. Now, to show that (4.4) is satisfied, we start noticing that

$$
\begin{aligned}
\partial(\operatorname{epi} B) \cap(C \times \mathbb{R}) & =\left\{(x, r) \in \mathbb{R}^{3}: x_{2}<0, r=0, x_{1} \leq \sqrt{-x_{2}}\right\} \cup\left\{(x, r) \in \mathbb{R}^{3}: x_{2}>0, r=1, x_{1} \leq \sqrt{x_{2}}\right\} \\
& \cup\left\{(x, r) \in \mathbb{R}^{3}: x_{2}=0,0 \leq r \leq 1\right\} .
\end{aligned}
$$

(See Fig. 3). That is, for each $x \in C$, thus $(x, B(x)) \in \partial(\operatorname{epi} B) \cap(C \times \mathbb{R})$, we have $\left[F(x) \cap T_{C}(x) \quad 0\right]^{\top} \subset$ $T_{\partial(\operatorname{epi} B) \cap(C \times \mathbb{R})}(x, B(x))$; hence, (4.4) follows using Proposition 3.2.3 of [2], the fact that

$$
\left[\partial_{P} B(x) \quad-1\right] \subset N_{\mathrm{epi} B \cap(C \times \mathbb{R})}^{P}(x, B(x)) \subset N_{\mathrm{epi} B \cap(C \times \mathbb{R})}(x, B(x)) \quad \forall x \in C,
$$


and since $\tilde{C} \subset C$ when $C$ is closed. Finally, in order to show that the function $B$ is not nonincreasing along solutions, we consider the function $\left(\phi(t), B\left(x_{o}\right)\right):=\left[\begin{array}{lll}t & t^{2} & 0\end{array}\right]^{\top} \in(C \times \mathbb{R})$ for all $t \geq 0$, which is absolutely continuous and solution to the differential equation $(\dot{x}, \dot{r})=\left(\left[\begin{array}{ll}1 & x_{1}\end{array}\right]^{\top}, 0\right) \in(F(x), 0)$.

To manage such a compromise, extra assumptions on the data $(C, F)$ of the system $\mathcal{H}_{f}$ need to be made.

\section{MAIN RESULTS}

In this section, we formulate necessary and sufficient infinitesimal conditions solving Problem 3.1 when the set $C$ in $\mathcal{H}_{f}$ given in (1.1) is not necessarily $\mathbb{R}^{n}$, not necessarily open, and nontrivial solutions are allowed to start from $\partial C$.

\subsection{When $B$ is lower semicontinuous and $\tilde{C}$ is generic}

The proposed approach, in this case, is based on transforming Problem 3.1 into the characterization of forward pre-invariance of a closed set for an augmented constrained differential inclusion, as described in the following lemma. The proof is in the appendix. Recall that the set $\tilde{C}$ is defined in (4.1).

Lemma 5.1. Consider a constrained differential inclusion $\mathcal{H}_{f}=(C, F)$. A lower semicontinuous function $B: \mathbb{R}^{n} \rightarrow \mathbb{R}$ satisfies ( $\star$ ) in Problem 3.1 if and only if the set epi $B \cap(\operatorname{cl}(C) \times \mathbb{R})$ is forward pre-invariant for the extended constrained differential inclusion in (1.2).

Proof. To prove necessity of epi $B \cap(\operatorname{cl}(C) \times \mathbb{R})$ being forward pre-invariant, we consider a nontrivial solution $(\phi, r): \operatorname{dom} \phi \rightarrow \mathbb{R}^{n+1}$ starting from $\left(x_{o}, r_{o}\right) \in \partial(\operatorname{epi} B \cap(\operatorname{cl}(C) \times \mathbb{R}))$ such that $t \mapsto B(\phi(t))$ is nonincreasing on $\operatorname{dom} \phi($ note that solutions from $\operatorname{int}(\operatorname{epi} B \cap(\operatorname{cl}(C) \times \mathbb{R}))$ reaching the boundary are already covered by this case). From the definition of the solutions to (1.1), we conclude that $\phi(t) \in \operatorname{cl}(C)$ for all $t \in \operatorname{dom} \phi$. So, to complete the proof of the necessary part, it is enough to show that $(\phi(t), r(t)) \in \operatorname{epi} B$ for all $t \in \operatorname{dom} \phi$. Indeed, $\left(x_{o}, r_{o}\right) \in \partial(\operatorname{epi} B \cap(\operatorname{cl}(C) \times \mathbb{R}))$ implies that either $\left(x_{o}, r_{o}\right) \in(\operatorname{int}(\operatorname{epi} B) \cap(\operatorname{cl}(C) \times \mathbb{R}))$, thus $r_{o}>B\left(x_{o}\right)$, or $\left(x_{o}, r_{o}\right) \in(\partial(\operatorname{epi} B) \cap(\operatorname{cl}(C) \times \mathbb{R}))$, thus $r_{o}=B\left(x_{o}\right)$. Hence, in both cases $r_{o} \geq B\left(x_{o}\right)$. Moreover, since $t \mapsto$ $B(\phi(t))$ is nonincreasing, it follows that $r_{o} \geq B(\phi(t))$ for all $t \in \operatorname{dom} \phi$ since $\phi(0)=x_{o} \in \partial C$. The latter fact implies that the solution $(\phi, r)$ satisfies $(\phi(t), r(t)) \in \operatorname{epi} B$ for all $t \in \operatorname{dom} \phi$ and necessity follows.

To prove the sufficient part, we use a contradiction argument. Suppose there exists $x_{o} \in \operatorname{cl}(C)$ and a nontrivial solution $\phi$ to $\mathcal{H}_{f}$ such that, for some $\epsilon>0, B(\phi(t))>B(\phi(0))$ for all $t \in(0, \epsilon]$. Since the set epi $B \cap(\operatorname{cl}(C) \times \mathbb{R})$ is forward pre-invariant, every solution $(\phi, r)$ starting from $\left(x_{o}, B\left(x_{o}\right)\right) \in \partial(\operatorname{epi} B) \cap(\operatorname{cl}(C) \times \mathbb{R})$ remains in $\operatorname{epi} B \cap(\operatorname{cl}(C) \times \mathbb{R})$ for all $t \in \operatorname{dom} \phi$. The latter fact implies that $\left(\phi(t), B\left(x_{o}\right)\right) \in \operatorname{epi} B$ for all $t \in \operatorname{dom} \phi$; hence, $B\left(x_{o}\right) \geq B(\phi(t))$ for all $t \in \operatorname{dom} \phi$, which yields a contradiction.

Forward pre-invariance has been extensively studied in the literature, see, e.g., $[2,13]$. Infinitesimal conditions for forward pre-invariance involving $F$ and tangent cones with respect to the considered closed set are shown to be necessary and sufficient when $C \equiv \mathbb{R}^{n}$. Our approach, in this case, is based on characterizing forward pre-invariance of the set epi $B \cap(\operatorname{cl}(C) \times \mathbb{R})$ using infinitesimal conditions.

Consider the following assumptions on the data $(C, F)$ of $\mathcal{H}_{f}$ :

(M1) For each $x_{o} \in \partial C \cap \tilde{C}$, if $F\left(x_{o}\right) \cap T_{C}\left(x_{o}\right) \neq \emptyset$ then, for each $v_{o} \in F\left(x_{o}\right) \cap T_{C}\left(x_{o}\right)$, there exist $U\left(x_{o}\right)-\mathrm{a}$ neighborhood of $x_{o}$ - and a continuous selection $v: \partial C \cap U\left(x_{o}\right) \rightarrow \mathbb{R}^{n}$ such that $v(x) \in F(x) \cap T_{C}(x)$ for all $x \in \partial C \cap U\left(x_{o}\right)$ and $v\left(x_{o}\right)=v_{o}$.

(M2) For each $x_{o} \in \partial C \cap \tilde{C}$, there exists $U\left(x_{o}\right)$ - a neighborhood of $x_{o}$ - such that $F(x) \subset T_{C}(x)$ for all $x \in U\left(x_{o}\right) \cap \partial C$. 
The need for (M1) and (M2) is discussed in Remarks 5.4 and 5.8. Furthermore, we consider the following condition:

$$
\langle\zeta, v\rangle \leq 0 \quad \forall\left[\zeta^{\top} \alpha\right]^{\top} \in N_{\mathrm{epi} B \cap(C \times \mathbb{R})}^{P}(x, B(x)), \quad \forall v \in F(x) \cap T_{C}(x), \quad \forall x \in \tilde{C} .
$$

The following result solves Problem 3.1. Its proof is inspired from Theorem 5.3.4 of [2] and Theorem 3.8 of [13].

Theorem 5.2. Consider a system $\mathcal{H}_{f}=(C, F)$ such that Assumption 2.5 holds and, additionally, $F$ is continuous. Let $B: \mathbb{R}^{n} \rightarrow \mathbb{R}$ be a lower semicontinuous function. Then,

1. $(\star)+(M 1) \Rightarrow(5.1)$.

2. F locally Lipschitz $+(5.1)+($ M2) $\Rightarrow(\star)$.

Consequently, when F is locally Lipschitz and (M1)-(M2) hold, (*) $\Leftrightarrow(5.1)$.

Proof. Using Lemma 5.1, items 2. and 1. in Theorem 5.2 follow if the following two statements are proved, respectively.

$1 \star$. The set epi $B \cap(\operatorname{cl}(C) \times \mathbb{R})$ is forward pre-invariant for (1.2) if (M2) and (5.1) hold.

$2 \star$. If the set epi $B \cap(\operatorname{cl}(C) \times \mathbb{R})$ is forward pre-invariant for (1.2) and (M1) holds, then (5.1) holds.

In order to prove item $2 \star$, we assume that the set epi $B \cap(\operatorname{cl}(C) \times \mathbb{R})$ is forward pre-invariant, that is, for each $\left(x_{o}, B\left(x_{o}\right)\right) \in \partial(\operatorname{epi} B) \cap(\tilde{C} \times \mathbb{R})$, each nontrivial solution starting from $\left(x_{o}, B\left(x_{o}\right)\right)$ remains in $\operatorname{epi} B \cap(\operatorname{cl}(C) \times \mathbb{R})$ along its entire domain. Furthermore, let us pick $v_{o} \in F\left(x_{o}\right) \cap T_{C}\left(x_{o}\right)$ and using (M1), we conclude the existence of a continuous selection $v: \partial C \cap U\left(x_{o}\right) \rightarrow \mathbb{R}^{n}$ such that $v(x) \in F(x) \cap T_{C}(x)$ for all $x \in \partial C \cap U\left(x_{o}\right)$ and $v\left(x_{o}\right)=v_{o}$. Moreover, since $F$ is continuous; thus, lower semicontinuous, and has closed and convex images, we use Michael's selection theorem [23] to conclude that the continuous selection $v$ on $\partial C \cap U\left(x_{o}\right)$ can be extended to a continuous selection $w: U\left(x_{o}\right) \rightarrow \mathbb{R}^{n}$ such that $w(x)=v(x)$ for all $x \in \partial C \cap U\left(x_{o}\right)$. Next, using Proposition 3.4 .2 of [2], we conclude the existence of a nontrivial solution $\phi$ starting from $x_{o}$ solution to the system $\dot{x}=w(x)$; thus, $\phi$ is also solution to $\mathcal{H}_{f}$ in (1.1). Next, since the set epi $B \cap(\operatorname{cl}(C) \times \mathbb{R})$ is forward pre-invariant, it follows that $t \mapsto\left(\phi(t), B\left(x_{o}\right)\right)$ is also a solution to (1.2) satisfying $\left(\phi(t), B\left(x_{o}\right)\right) \subset \operatorname{epi} B \cap(\operatorname{cl}(C) \times \mathbb{R})$ for all $t \in \operatorname{dom} \phi$. Furthermore, consider a sequence $\left\{t_{i}\right\}_{i=0}^{\infty} \rightarrow 0$ and let $v_{i}:=\frac{\phi\left(t_{i}\right)-\phi(0)}{t_{i}}$. Now, since $\phi$ is solution to $\dot{x}=w(x)$ and $w$ is continuous, it follows that $\lim _{i \rightarrow \infty} v_{i}=v_{o}$. At the same time, having $\left(\phi\left(t_{i}\right), B\left(x_{o}\right)\right)=\left(\left(\phi(0)+v_{i} t_{i}\right), B\left(x_{o}\right)\right) \in$ epi $B \cap(C \times \mathbb{R})$ and using the equivalence (see [4], P. 122)

$$
v \in T_{K}(x) \Longleftrightarrow \exists\left\{h_{i}\right\}_{i \in \mathbb{N}} \rightarrow 0^{+} \text {and }\left\{v_{i}\right\}_{i \in \mathbb{N}} \rightarrow v: x+h_{i} v_{i} \in K,
$$

we conclude that $\left[\begin{array}{ll}v_{o}^{\top} & 0\end{array}\right]^{\top} \in T_{\mathrm{epi} B \cap(C \times \mathbb{R})}\left(x_{o}, B\left(x_{o}\right)\right)$. Hence, using ${ }^{4}$ ([2], Prop. 3.2.3), we conclude that for each $\left[\begin{array}{ll}\zeta^{\top} & \alpha\end{array}\right]^{\top} \in N_{\mathrm{epi} B \cap(C \times \mathbb{R})}^{P}\left(x_{o}, B\left(x_{o}\right)\right),\left[\zeta^{\top} \alpha\right]^{\top} \in N_{\mathrm{epi} B \cap(C \times \mathbb{R})}\left(x_{o}, B\left(x_{o}\right)\right)$. Thus, (5.1) by definition of the normal cone $N_{\text {epi } B \cap(C \times \mathbb{R})}$.

Next, we prove item $1 \star$ using contradiction. Indeed, we consider $t_{1}>0$ such that a solution $z$ to (1.2) starting from $z_{a o}:=\left(x_{o}, B\left(x_{o}\right)\right) \in \partial($ epi $B) \cap(\tilde{C} \times \mathbb{R})$ satisfies $z_{a}(t):=\left(x(t), B\left(x_{o}\right)\right) \in(\operatorname{cl}(C) \times \mathbb{R}) \backslash$ epi $B$ for all $t \in\left(0, t_{1}\right)$ and such that

$$
\dot{z}_{a}(t) \in F_{a}\left(z_{a}(t)\right):=\left[F(x(t))^{\top} \quad 0\right]^{\top} \quad \text { for a.a. } t \in\left(0, t_{1}\right)
$$

\footnotetext{
${ }^{4}$ With $x=\left(x_{o}, B\left(x_{o}\right)\right), v=\left[v^{\top} \quad 0\right]^{\top}, K=\operatorname{epi} B \cap(C \times \mathbb{R})$, and $N_{K}^{0}=N_{K}$.
} 
Furthermore, for $t \in\left[0, t_{1}\right)$, we use $y(t)$ to denote the projection of $z_{a}(t)$ on the set $\operatorname{epi} B \cap(\operatorname{cl}(C) \times \mathbb{R})$ and we define

$$
\delta(t):=\left|z_{a}(t)-y(t)\right| \quad \forall t \in\left[0, t_{1}\right) .
$$

That is, by construction, we have $\delta(0)=0$ and $\delta(t)>0$ for all $t \in\left(0, t_{1}\right)$ for $t_{1}$ sufficiently small. Now, using the fact that

$$
\delta(t+h)=\left|z_{a}(t+h)-y(t+h)\right| \leq\left|z_{a}(t+h)-y(t)\right|,
$$

plus the identity $a-b=\left(a^{2}-b^{2}\right) /(a+b)$ for $a$ and $b \geq 0$, we derive the following inequality for some $h>0$ sufficiently small

$$
\delta(t+h)-\delta(t) \leq\left|z_{a}(t+h)-y(t)\right|-\left|z_{a}(t)-y(t)\right|=\frac{\left|z_{a}(t+h)-y(t)\right|^{2}-\left|z_{a}(t)-y(t)\right|^{2}}{\left|z_{a}(t+h)-y(t)\right|+\left|z_{a}(t)-y(t)\right|} .
$$

Furthermore, assume that $t$ is chosen such that $\dot{z}_{a}(t)$ exists. Hence, we can replace $z_{a}(t+h)$ by

$$
z_{a}(t+h)=z_{a}(t)+h \dot{z}_{a}(t)+o(h),
$$

where $o(h)$ is the remainder of the first order Taylor expansion of $h \mapsto z_{a}(t+h)$ around $h=0$, which satisfies $\lim _{h \rightarrow 0} o(h) / h=0$. Furthermore, using the inequality

$$
\left|z_{a}(t+h)-y(t)\right| \geq\left|z_{a}(t)-y(t)\right|-h\left|\dot{z}_{a}(t)+o(h) / h\right|,
$$

we conclude that the denominator in (5.3) satisfies

$$
\left|z_{a}(t+h)-y(t)\right|+\left|z_{a}(t)-y(t)\right| \geq 2\left|z_{a}(t)-y(t)\right|-h\left|\dot{z}_{a}(t)+o(h) / h\right|
$$

while the numerator in (5.3) is upper bounded by

$$
2 \dot{z}_{a}(t)^{\top}\left(z_{a}(t)-y(t)\right)+h^{2}\left|\dot{z}_{a}(t)+o(h) / h\right|^{2}+2 o(h)^{\top}\left(z_{a}(t)-y(t)\right) .
$$

Hence, letting $h \rightarrow 0^{+}$we obtain,

$$
\limsup _{h \rightarrow 0^{+}} \frac{\delta(t+h)-\delta(t)}{h} \leq \frac{\left(z_{a}(t)-y(t)\right)^{\top} \dot{z}_{a}(t)}{\left|z_{a}(t)-y(t)\right|} .
$$

We have the following claim.

Claim 5.3. Under (5.1), (M2), and when $t>0$ is sufficiently small, the following holds:

$$
\left(z_{a}(t)-y(t)\right)^{\top} \eta_{y} \leq 0 \quad \forall \eta_{y} \in F_{a}(y(t))
$$

Under (5.6) and for each $\eta_{y} \in F_{a}(y(t))$, the inequality (5.5) can be re-written as

$$
\limsup _{h \rightarrow 0^{+}} \frac{\delta(t+h)-\delta(t)}{h} \leq \frac{\left(z_{a}(t)-y(t)\right)^{\top}\left[\dot{z}_{a}(t)-\eta_{y}\right]}{\left|z_{a}(t)-y(t)\right|} .
$$


Since $\dot{z}_{a}(t) \in F_{a}\left(z_{a}(t)\right)$, using the fact that the map $F_{a}$ is locally Lipschitz, it is always possible to find a constant $\gamma>0$ and $\eta_{y}^{*} \in F(y(t))$ such that, when replacing $\eta_{y}$ by $\eta_{y}^{*}$ in (5.7), we obtain, for almost all $t \in\left(0, t_{1}\right)$,

$$
\limsup _{h \rightarrow 0^{+}} \frac{\delta(t+h)-\delta(t)}{h} \leq \gamma\left|z_{a}(t)-y\right|=\gamma \delta(t)
$$

The contradiction follows since (5.8) implies that $\delta(t)=0$ for all $t \in\left(0, t_{1}\right)$ due to $\delta(0)=0$ by construction.

Proof of Claim 5.3: To prove the latter claim, we start noticing, using the continuity of the system's solutions, that for any neighborhood sufficiently small around $z_{a o}$ denoted $U\left(z_{a o}\right)$, there exists $t_{1}>0$ sufficiently small such that $y(t) \in U\left(z_{a o}\right)$ for all $t \in\left[0, t_{1}\right)$. Furthermore, under (M2) and for $U\left(z_{a o}\right)$ small enough, we show that $y(t)$ either belongs to $\partial(\operatorname{epi} B) \cap(\tilde{C} \times \mathbb{R})$ or to the set $\operatorname{int}($ epi $B) \cap(\partial C \times \mathbb{R})$. Indeed, by definition of the projection, $y(t)$ cannot belong to $\operatorname{int}(\operatorname{epi} B) \cap(\operatorname{int}(C) \times \mathbb{R})=\operatorname{int}(\operatorname{epi} B \cap(C \times \mathbb{R}))$. Furthermore, (M2) implies that, when $U\left(z_{a o}\right)$ is small enough, a nontrivial solution starting from $y(t)$ always exists; hence, $y(t) \in \tilde{C} \times \mathbb{R}$. Now, we consider the two possibilities of $y(t)$.

- When $y(t) \in \partial(\operatorname{epi} B) \cap(\tilde{C} \times \mathbb{R}),(5.6)$ follows from (5.1). Indeed, by definition of $N_{\text {epi } B \cap(C \times \mathbb{R})}^{P}$, we conclude that $z_{a}(t)-y(t) \in N_{\mathrm{epi} B \cap(C \times \mathbb{R})}^{P}(y(t))$. Furthermore, using (M2) for $t>0$ small enough, we conclude that $F_{a}(y(t)) \subset T_{\operatorname{epi} B \cap(C \times \mathbb{R})}(y(t))$.

- When $y(t) \in \operatorname{int}(\operatorname{epi} B) \cap(\partial C \times \mathbb{R})$, since $y(t) \in U\left(z_{a o}\right)$, it follows using (M2) that $F_{a}(y(t)) \subset T_{C}(y(t))$. Furthermore, since $y(t) \in \operatorname{int}(\operatorname{epi} B)$, it follows that $F_{a}(y(t)) \subset T_{\text {epi } B \cap(C \times \mathbb{R})}(y(t))$.

Now, in order to conclude (5.6), for each $\eta_{y} \in F_{a}(y(t))$, we introduce the inequality, for some $h>0$,

$$
\left|z_{a}(t)-y(t)\right| \leq\left|z_{a}(t)-y(t)-h \eta_{y}\right|+\left|y(t)+h \eta_{y}\right|_{K}
$$

where $K:=\operatorname{epi} B \cap(C \times \mathbb{R})$. To obtain the previous inequality, we used the fact that $|\cdot|_{K}$ is globally Lipschitz with Lipschitz constant equal to 1 and $\left|z_{a}(t)-y(t)\right|=\left|z_{a}(t)\right|_{K}$. Next, by taking the square in both sides of inequality (5.9) and dividing by $h$, we obtain, for each $\eta_{y} \in F_{a}(y(t))$,

$$
\begin{aligned}
& \frac{\left|z_{a}(t)-y(t)\right|^{2}}{h} \leq \frac{\left|z_{a}(t)-y(t)-h \eta_{y}\right|^{2}}{h}+\frac{\left|y(t)+h \eta_{y}\right|_{K}^{2}}{h}+\frac{2\left|z_{a}(t)-y(t)-h \eta_{y}\right|\left|y(t)+h \eta_{y}\right|_{K}}{h} \\
\leq & \frac{\left|z_{a}(t)-y(t)\right|^{2}}{h}+h\left|\eta_{y}\right|^{2}-2\left(z_{a}(t)-y(t)\right) \eta_{y}+h\left(\frac{\left|y(t)+h \eta_{y}\right|_{K}}{h}\right)^{2}+\frac{2\left|z_{a}(t)-y(t)-h \eta_{y}\right|\left|y(t)+h \eta_{y}\right|_{K}}{h} .
\end{aligned}
$$

Finally, letting $h \rightarrow 0^{+}$through a suitable sequence, (5.6) is proven using the fact that $\lim \inf _{h \rightarrow 0^{+}} \frac{\left|y(t)+h \eta_{y}\right|_{K}}{h}=$ 0 since we already have $\eta_{y} \in T_{K}(y(t))$.

Remark 5.4. Condition (M1) ensures the existence of a nontrivial solution (i.e., solution whose domain is not a singleton) along each direction in the intersection between the images of $F$ and the contingent cone $T_{C}$. The latter requirement is necessary in order to prove the necessary part of the statement in the general case where $C$ is not $\mathbb{R}^{n}$ and nontrivial solutions start from $\partial C$, see Example 4.2.

In other words, when $t \mapsto B(\phi(t))$ is nonincreasing along the nontrivial solutions to $\mathcal{H}_{f}$, showing that, for each $x_{o} \in \tilde{C}$, every $v_{o} \in F\left(x_{o}\right) \cap T_{C}\left(x_{o}\right)$ satisfies (5.1) naturally imposes the existence of at least one nontrivial solution starting from $x_{o}$ that is tangent to $v_{o}$ at $x_{o}$.

Remark 5.5. The existence of a nontrivial solution along each direction within the set $F\left(x_{o}\right)$ holds for free when $x_{o} \in \operatorname{int}(C)$. Indeed, in this case, for each $v_{o} \in F\left(x_{o}\right) \cap T_{C}\left(x_{o}\right)=F\left(x_{o}\right)$, there exists a selection $v: U\left(x_{o}\right) \rightarrow \mathbb{R}^{n}$, $U\left(x_{o}\right) \subset C$, such that $v(x) \in F(x)$ for all $x \in U\left(x_{o}\right)$ and $v\left(x_{o}\right)=v_{o}$. The latter selection can be chosen to be continuous when $F$ is locally Lipschitz. Hence, the differential equation $\dot{x}=v(x)$ admits a continuously 
differentiable solution $\phi$ starting from $x_{o}$ with $\dot{\phi}(0)=v_{o}$, which is also solution to $\mathcal{H}_{f}$; thus, the solution $\phi$ is tangent to $v_{o}$ at $x_{o}$.

Remark 5.6. The Assumption (M1) holds for free for example if $C$ is closed, $\operatorname{dom}\left(F \cap T_{C}\right) \cap \partial C$ is open relative to $\partial C, F \cap T_{C}$ is lower semicontinuous at least on $\operatorname{dom}\left(F \cap T_{C}\right) \cap \partial C$, and the set $C$ is regular. Indeed, having $T_{C}(x)=C_{C}(x)$ for all $x \in \operatorname{cl}(C)$ implies that $T_{C}$ is convex for all $x \in \operatorname{cl}(C)$ and the same holds for $F(x) \cap T_{C}(x)$ since $F(x)$ is also convex for all $x \in C$. Hence, a direct application of Michæl's Theorem ([23], Thm. 3.2) to the set-valued map $F \cap T_{C}$ defined on the open set $\operatorname{dom}\left(F \cap T_{C}\right) \cap \partial C$ relative to $\partial C$, (M1) follows.

Remark 5.7. Assumption (M1) can be replaced by the following relaxed assumption involving some extra knowledge concerning the system's solutions.

(M1') For each $x_{o} \in \partial C \cap \widetilde{C}$ and for each $v_{o} \in F\left(x_{o}\right) \cap T_{C}\left(x_{o}\right)$, there exists a solution $\phi:[0, h] \mapsto \phi(t)$, for some $h>0$, starting from $x_{o}$ and a sequence $\left\{h_{i}\right\}_{i=0}^{\infty} \rightarrow 0$ and $\lim _{i \rightarrow \infty}\left(\phi\left(h_{i}\right)-x_{o}\right) / h_{i}=v_{o}$.

Remark 5.8. When (M2) is not satisfied, as shown in Example 4.3, there exist situations where the statement of Theorem 5.2 does not hold even if all the remaining conditions therein are satisfied. As a consequence, constraining more vector fields rather than only those in $F(x) \cap T_{C}(x)$, as proposed in (5.1), is important to prove the sufficient part in Theorem 5.2. However, strengthening (5.1) would affect the statement in 2., the reason why a global assumption similar to (M2) that is independent from the function $B$ must be considered.

In the following, we show how Theorem 5.2 applies to solve Problem 3.1 on a concrete example.

Example 5.9. The continuous dynamics of the bouncing-ball hybrid model is given by $\mathcal{H}_{f}:=(C, F)$ with $F(x):=\left[\begin{array}{ll}x_{2} & -\gamma\end{array}\right]^{\top}$ for all $x \in C:=\left\{x \in \mathbb{R}^{2}: x_{1} \geq 0\right\}$. The constant $\gamma>0$ is the gravitational acceleration. First, $F$ is single valued and continuously differentiable; hence, Assumption 2.5 holds. Second, note that $\widetilde{C}:=C \backslash\left\{x \in \mathbb{R}^{2}: x_{1}=0, x_{2} \leq 0\right\}$. Hence, starting from $x_{o} \in \widetilde{C} \cap \partial C=\left\{x \in \mathbb{R}^{2}: x_{1}=0, \quad x_{2}>0\right\}$, $F\left(x_{o}\right)=\left[\begin{array}{ll}x_{o 2} & -\gamma\end{array}\right]^{\top} \in T_{C}\left(x_{o}\right)$; thus, (M2) follows. Moreover, (M1) is also satisfied since $\partial C \cap \widetilde{C}$ is open and, for each $x_{o} \in \partial C \cap \widetilde{C}, F\left(x_{o}\right) \in T_{C}\left(x_{o}\right)$. Finally, using Theorem 5.2, we conclude that a lower semicontinuous function $B: \mathbb{R}^{2} \rightarrow \mathbb{R}$ satisfies $(\star)$ if and only if (5.1) holds. In particular, the energy function of the bouncing ball satisfies (5.1) since, by definition, it cannot increase along the solutions.

The following result shows that, in some situations, (M1) is not needed. However, such situations require that the set $\widetilde{C}$ and the function $B$ satisfy the following extra assumptions:

Assumption 5.10. $x \mapsto \operatorname{blckdiag}\left\{I_{n}, 0\right\} N_{\text {epi } B \cap(C \times \mathbb{R})}^{P}(x, B(x))$ is lower semicontinuous on $\widetilde{C}$.

Assumption 5.11. $U(x) \cap \operatorname{int}(C) \neq \emptyset$ for all $x \in \partial C \cap C$ and for all $U(x)$.

Theorem 5.12. Consider a system $\mathcal{H}_{f}=(C, F)$ such that Assumption 2.5 holds and $F$ is additionally continuous. Let $B: \mathbb{R}^{n} \rightarrow \mathbb{R}$ be a lower semicontinuous function. Then,

$-(\star)+$ Assumption $5.10+$ Assumption $5.11 \Rightarrow(5.1)$.

Consequently, when F is locally Lipschitz and (M2) and Assumptions 5.10-5.11 hold, ( $*$ ) $\Leftrightarrow(5.1)$.

Proof. We distinguish two situations.

- First, we consider the case where $x \in \operatorname{int}(C)$. In this case, according to Remark 5.5, (M1) holds trivially. Hence, using Theorem 5.2, we conclude that

$$
\langle\zeta, v\rangle \leq 0 \quad \forall\left[\zeta^{\top} \alpha\right]^{\top} \in N_{\mathrm{epi} B \cap(C \times \mathbb{R})}^{P}(x, B(x)), \quad \forall v \in F(x) \cap T_{C}(x) .
$$

- Next, when $x \in \widetilde{C} \backslash \operatorname{int}(C)$, we show that (5.11) holds using a contradiction. That is, let us assume the existence of $\left[\begin{array}{ll}\zeta_{o}^{\top} & \alpha_{o}\end{array}\right]^{\top} \in N_{\mathrm{epi} B \cap(C \times \mathbb{R})}^{P}(x, B(x))$ and $v_{o} \in F(x) \cap T_{C}(x)$ such that $\left\langle\zeta_{o}, v_{o}\right\rangle>0$; thus, 
$\left\langle\zeta_{o}, v_{o}\right\rangle \geq \epsilon$ for some $\epsilon>0$. Next, using the continuity of $F$ both with Assumption 5.11, we conclude that for each $\epsilon_{1}>0$ there exist $U(x)$ such that for each $y \in U(x) \cap \operatorname{int}(C)(U(x) \cap \operatorname{int}(C) \neq \emptyset$ under Assumption 5.11) there exists $v_{y} \in F(y)$ such that $\left|v_{y}-v_{o}\right| \leq \epsilon_{1}$. On the other hand, using Assumption 5.10 under Assumption 5.11, we conclude the existence of $x_{1} \in U(x) \cap \operatorname{int}(C)$ sufficiently close to $x$ and $\left[\begin{array}{ll}\zeta_{1} & \alpha_{1}\end{array}\right]^{\top} \in N_{\mathrm{epi} B \cap(C \times \mathbb{R})}^{P}\left(x_{1}, B\left(x_{1}\right)\right)$ such that $\left|\zeta_{1}-\zeta_{o}\right| \leq \epsilon_{1}$. Furthermore, since $x_{1} \in \operatorname{int}(C)$, using the first part of the proof, we conclude that $\left\langle\zeta_{1}, v_{x_{1}}\right\rangle \leq 0$. However, since $\left\langle\zeta_{o}, v_{o}\right\rangle \geq \epsilon$ it follows that

$$
\left\langle\zeta_{o}, v_{o}\right\rangle=\left\langle\zeta_{1}, v_{x_{1}}\right\rangle+\left\langle\left(\zeta_{o}-\zeta_{1}\right), v_{o}\right\rangle+\left\langle\zeta_{o},\left(v_{o}-v_{x_{1}}\right)\right\rangle+\left\langle\left(\zeta_{1}-\zeta_{o}\right),\left(v_{o}-v_{x_{1}}\right)\right\rangle \geq \epsilon .
$$

Hence, $\left\langle\zeta_{1}, v_{x_{1}}\right\rangle \geq \epsilon-\left(\left|v_{o}\right|+\left|\zeta_{o}\right|\right) \epsilon_{1}-\epsilon_{1}^{2}$. Finally, taking $\epsilon_{1}=\min \left\{\frac{\epsilon}{2\left(\left(\left|v_{o}\right|+\left|\zeta_{o}\right|\right)+1\right)}, 1\right\}$, the contradiction follows since the latter implies that $\left\langle\zeta_{1}, v_{x_{1}}\right\rangle \geq \epsilon / 2>0$.

In the sequel, we will show that the inequality in (5.1) does not need to be checked for all $\left[\begin{array}{ll}\zeta^{\top} & \alpha\end{array}\right]^{\top} \in$ $N_{\text {epi } B \cap(C \times \mathbb{R})}^{P}(x, B(x))$ when $x \in \operatorname{int}(C)$. That is, when $x \in \operatorname{int}(C)$, we will show that it is enough to verify the inequality in (5.1) only for the vectors $\left[\zeta^{\top} \alpha\right]^{\top} \in N_{\mathrm{epi} B \cap(\operatorname{cl}(C) \times \mathbb{R})}^{P}(x, B(x))$ with $\alpha=-1$ to conclude that it holds for all $\left[\begin{array}{ll}\zeta^{\top} & \alpha\end{array}\right]^{\top} \in N_{\mathrm{epi} B \cap(C \times \mathbb{R})}^{P}(x, B(x))$. The former subset is generated by the proximal subdifferential $\partial_{P} B$ introduced in (3.12). Although $\partial_{P} B$ can fail to exist at some points $(x, B(x)) \in \operatorname{epi} B \cap(\operatorname{int}(C) \times \mathbb{R})$, its density property in Lemma A.1 in the Appendix is enough to preserve the equivalence in Theorem 5.2.

Proposition 5.13. Consider a system $\mathcal{H}_{f}=(C, F)$ such that Assumption 2.5 holds, $F$ is additionally locally Lipschitz, and let $B: \mathbb{R}^{n} \rightarrow \mathbb{R}$ be a lower semicontinuous function. Then, (5.11) is satisfied at $x \in$ int $(C)$ if

$$
\langle\zeta, \eta\rangle \leq 0 \quad \forall \zeta \in \partial_{P} B(x), \quad \forall \eta \in F(x)
$$

Proof. Let $x \in \operatorname{int}(C)$ and let $\left[\zeta^{\top} \quad 0\right]^{\top} \in N_{S}^{P}(x, B(x))$. After Lemma A.1, we conclude the existence of a sequence $\left(x_{i}, \epsilon_{i}, \zeta_{i}\right) \in \operatorname{int}(C) \times \mathbb{R}_{>0} \times \partial_{P} B\left(x_{i}\right)$ such that $\left(x_{i}, \epsilon_{i}, \zeta_{i}\right) \rightarrow(x, 0, \zeta)$ and $\left[\zeta_{i}^{\top} \quad-\epsilon_{i}\right]^{\top} \in$ $N_{\text {epi } B}^{P}\left(x_{i}, B\left(x_{i}\right)\right)$. Moreover, since $F$ is locally Lipschitz, it follows that, for each $\eta_{x} \in F(x)$, there exists $v_{i} \in F\left(x_{i}\right)$ such that $\left|v_{i}-\eta_{x}\right| \leq K\left|x_{i}-x\right|$ for some $K>0$. According to (5.12), we conclude that, for all $i \in \mathbb{N}$,

$$
\left\langle\zeta_{i}, v_{i}\right\rangle=\left\langle\zeta, \eta_{x}\right\rangle+\left\langle\zeta_{i}, v_{i}-\eta_{x}\right\rangle+\left\langle\zeta_{i}-\zeta, \eta_{x}\right\rangle \leq 0
$$

Finally, on the limit we conclude that $\left\langle\zeta, \eta_{x}\right\rangle \leq 0$ for all $\eta_{x} \in F(x)$.

\subsection{When $B$ is lower semicontinuous and $\operatorname{cl}(C)$ is pre-contractive}

When the set $C \equiv \mathbb{R}^{n}$, or when $\widetilde{C}$ is open, the following necessary and sufficient infinitesimal condition solving Problem 3.1 is provided in Theorem 6.3 of [13].

$$
\langle\zeta, \eta\rangle \leq 0 \quad \forall \zeta \in \partial_{P} B(x), \quad \forall \eta \in F(x), \quad \forall x \in \operatorname{int}(C) .
$$

In the following statement, we recover ([13], Thm. 6.3) as a direct consequence of Theorem 5.2 and Proposition 5.13. Furthermore, as we will show, Condition (5.13) can also be used when the following extra assumptions hold.

Assumption 5.14. The set $\operatorname{cl}(C)$ is pre-contractive.

Assumption 5.15. $B$ is continuous on $\partial C \cap \widetilde{C}$.

Corollary 5.16. Consider a system $\mathcal{H}_{f}=(C, F)$ such that Assumption 2.5 holds and $F$ is additionally continuous. Let $B: \mathbb{R}^{n} \rightarrow \mathbb{R}$ be a lower semicontinuous function. Then, 
1. $(*) \Rightarrow(5.13)$.

2. When $F$ is locally Lipschitz and either $\widetilde{C}$ is open or Assumptions $5.14-5.15$ hold, $(\star) \Leftrightarrow(5.13)$.

Proof. The proof of item 1 follows from the first item in Theorem 5.2 since (M1) holds trivially when $x_{o} \in \operatorname{int}(C)$, see Remark 5.5.

The proof of item 2, when $\widetilde{C}$ is open, follows from a direct application of Theorem 5.2 and Proposition 5.13. Indeed, we notice that when $\widetilde{C}$ is open, (M1) and (M2) hold trivially because $\partial C \cap \widetilde{C}=\emptyset$. Now, we assume that Assumptions 5.14-5.15 hold. Using the previous step, we conclude that, along the solutions $\phi: \operatorname{dom} \phi \rightarrow \operatorname{int}(C)$, $t \mapsto B(\phi(t))$ is nonincreasing if and only if (5.12) holds. To complete the proof, we will show that, under Assumptions 5.14-5.15, if $t \mapsto B(\phi(t))$ is nonincreasing along the solutions $\phi: \operatorname{dom} \phi \rightarrow \operatorname{int}(C)$ then so it is along the solutions $\phi: \operatorname{dom} \phi \rightarrow \operatorname{cl}(C)$ using contradiction. Consider a solution $\phi: \operatorname{dom} \phi \rightarrow \operatorname{cl}(C)$ such that $t \mapsto B(\phi(t))$ fails to be nonincreasing. Since $\phi$ cannot flow in $\partial C$ under Assumption 5.14, $t \mapsto B(\phi(t))$ is nonincreasing in the interior $C$, and since $B$ is only lower semicontinuous, for the map $t \mapsto B(\phi(t))$ to fail to be nonincreasing either one of the following holds: For some $\epsilon>0, B(\phi(0))<B(\phi(t))$ for all $t \in(0, \epsilon]$, or, for some $T>0$ such that $\phi(T) \in \partial C, B(\phi(T))>B(\phi(T-t))$ for all $t \in(0, \epsilon]$. The latter scenario contradicts the lower semicontinuity of $B$, and the first one contradicts Assumption 5.15.

Before closing this section, the following remark is in order.

Remark 5.17. It is important to notice that, when $x \in \partial C \cap \widetilde{C}$, we need to impose a condition similar to (5.1) since the relaxed condition in (5.12) is not enough to guarantee the equivalence. Indeed, when $x \in \operatorname{int}(C)$, (5.12) indicates that the inequality therein holds for all $\left[\begin{array}{ll}\zeta & -1\end{array}\right]^{\top} \in N_{\operatorname{epi} B \cap(C \times \mathbb{R})}^{P}(x, B(x))$, moreover, when $\left[\begin{array}{ll}\zeta & 0\end{array}\right]^{\top} \in N_{\mathrm{epi} B \cap(C \times \mathbb{R})}^{P}(x, B(x))$, it is possible to show that the inequality in (5.12) remains satisfied using Lemma A.1. Hence, using Lemma A.1, we can find a point in any neighborhood of $U(x)$ such that (5.12) holds. The latter fact is not necessarily true when $x \in \partial C \cap \widetilde{C}$, since the points in the neighborhood of $x$ are not necessarily in $\widetilde{C}$; thus, there is no guarantee to find a point within any neighborhood of $x$ such that (5.12) holds.

\subsection{When $B$ is locally Lipschitz and $\tilde{C}$ is generic}

In this case, we show that (M2) is not required. Indeed, such a relaxation is possible since the generalized gradient $\partial_{C} B$ introduced in Definition 3.3 will be used instead of the proximal subdifferential. Thanks to Lebourg's mean-value Theorem in Lemma A.3 and to the lower Dini-derivative-based condition in Lemma 3.2, which combined together provide a useful relation between the lower Dini derivative of $B$ along the system's solutions and the generalized gradient $\partial_{C} B$.

In this section, we consider the following infinitesimal conditions:

$$
\begin{array}{ll}
\langle\eta, \zeta\rangle \leq 0 \quad \forall \eta \in \partial_{C} B(x), \quad \forall \zeta \in F(x) \cap T_{C}(x), \quad \forall x \in \widetilde{C} . \\
\langle\eta, \zeta\rangle \leq 0 \quad \forall \eta \in \partial_{C} B(x), \quad \forall \zeta \in F(x) \cap T_{C}(x): \exists c \in \mathbb{R}:\langle\eta, \zeta\rangle=c \text { for all } \eta \in \partial_{C} B(x), \quad \forall x \in \widetilde{C} .
\end{array}
$$

Furthermore, we recall from [30] the following notion of nonpathological functions.

Definition 5.18. A locally Lipschitz function $B: \mathbb{R}^{n} \rightarrow \mathbb{R}$ is nonpathological if, for each absolutely continuous function $\phi: \operatorname{dom} \phi \rightarrow \mathbb{R}^{n}, \operatorname{dom} \phi \subset \mathbb{R}$, the set $\partial_{C} B(\phi(t))$ is a subset of an affine subspace orthogonal to $\dot{\phi}(t)$ for almost all $t \in \operatorname{dom} \phi$. Namely, for almost all $t \in \operatorname{dom} \phi$, there exists $a_{t} \in \mathbb{R}$ such that

$$
\langle\eta, \dot{\phi}(t)\rangle=a_{t} \quad \forall \eta \in \partial_{C} B(\phi(t)) .
$$

Remark 5.19. Using Theorem 4 of [30], we conclude that locally Lipschitz and regular functions are nonpathological functions. In addition, locally Lipschitz functions that are semiconcave or semiconvex are nonpathological - in particular, finite-valued convex functions are nonpathological.

Now, we are ready to provide our characterization of $(\star)$ when $B$ is locally Lipschitz. 
Theorem 5.20. Consider a system $\mathcal{H}_{f}=(C, F)$ such that Assumption 2.5 holds. Let $B: \mathbb{R}^{n} \rightarrow \mathbb{R}$ be a locally Lipschitz function. Then,

1. $(5.14) \Rightarrow(\star)$.

2. When (M1) holds, $F$ is continuous, and $B$ is regular, $(\star) \Leftrightarrow(5.14)$.

3. When $B$ is nonpathological, $(5.15) \Rightarrow(*)$.

Proof. We prove item 1 using contradiction. That is, consider a nontrivial solution $\phi:[0, T] \rightarrow \operatorname{cl}(C), T>0$, such that the function $t \mapsto B(\phi(t))$ is strictly increasing on $[0, T]$. That is, using Lemma 3.2, it follows that, for each $t_{o} \in[0, T)$, we have

$$
\liminf _{t \rightarrow t_{o}^{+}} \frac{B(\phi(t))-B\left(\phi\left(t_{o}\right)\right)}{t-t_{o}} \geq \epsilon>0
$$

Next, using Lemma A.3, we conclude that, for each $t \in\left[t_{o}, T\right]$, there exists $u_{t}$ belonging to the open line segment $\left(\phi\left(t_{o}\right), \phi(t)\right)$ such that

$$
\frac{B(\phi(t))-B\left(\phi\left(t_{o}\right)\right)}{t-t_{o}} \in\left\{\left\langle z, \frac{\phi(t)-\phi\left(t_{o}\right)}{t-t_{o}}\right\rangle: z \in \partial_{C} B\left(u_{t}\right)\right\}
$$

Hence, there exists $w_{t} \in \partial_{C} B\left(u_{t}\right)$ such that

$$
\frac{B(\phi(t))-B\left(\phi\left(t_{o}\right)\right)}{t-t_{o}}=\left\langle w_{t}, \frac{\phi(t)-\phi\left(t_{o}\right)}{t-t_{o}}\right\rangle .
$$

Furthermore, (5.16) implies the existence of a sequence $\left\{t_{n}\right\}_{n=0}^{\infty} \subset\left(t_{o}, T\right]$ with $t_{n} \rightarrow t_{o}$ such that

$$
\lim _{n \rightarrow \infty} \frac{B\left(\phi\left(t_{n}\right)\right)-B\left(\phi\left(t_{o}\right)\right)}{t_{n}-t_{o}}=\lim _{n \rightarrow \infty}\left\langle w_{t_{n}}, \frac{\phi\left(t_{n}\right)-\phi\left(t_{o}\right)}{t_{n}-t_{o}}\right\rangle \geq \epsilon>0 .
$$

Now, since $\partial_{C} B$ is locally bounded, there exist $U\left(\phi\left(t_{o}\right)\right)$ and $K>0$ such that $|\zeta| \leq K$ for all $\zeta \in \partial_{C} B(x)$ and for all $x \in U\left(\phi\left(t_{o}\right)\right)$. Furthermore, since the system's solutions are continuous, it follows that for $T$ sufficiently small, both $\phi(t)$ and $u_{t}$ belong to $U\left(\phi\left(t_{o}\right)\right)$ for all $t \in\left[t_{o}, T\right]$. Hence,

$$
\left|w_{t_{n}}\right| \leq K \quad \forall n \in \mathbb{N} .
$$

Similarly, since $F$ is locally bounded, then, there exists $U\left(\phi\left(t_{o}\right)\right)$ and $K>0$ such that $|y| \leq K$ for all $y \in F(x)$ and for all $x \in U\left(\phi\left(t_{o}\right)\right)$. Furthermore, since the system's solutions are continuous, it follows that for $T>0$ sufficiently small, $\phi(s) \in U\left(\phi\left(t_{o}\right)\right)$ for all $s \in\left[t_{o}, T\right]$. Hence, in view of the integral

$$
\phi(t)-\phi\left(t_{o}\right)=\int_{t_{o}}^{t} \dot{\phi}(s) d s \quad \dot{\phi}(s) \in F(\phi(s)) \quad \text { for a.a. } \quad s \in\left[t_{o}, t\right] \text { and } \quad \forall t \in\left[t_{o}, T\right]
$$

we conclude that

$$
\left|\frac{\phi\left(t_{n}\right)-\phi\left(t_{o}\right)}{t_{n}-t_{o}}\right| \leq K \quad \forall n \in \mathbb{N}
$$


By passing to a subsequence, we conclude the existence of $w_{o} \in \mathbb{R}^{n}$ and $v_{o} \in \mathbb{R}^{n}$ such that

$$
w_{t_{n}} \rightarrow w_{o} \quad \text { and } \quad \frac{\phi\left(t_{n}\right)-\phi\left(t_{o}\right)}{t_{n}-t_{o}} \rightarrow v_{o}
$$

Furthermore, since $w_{t_{n}} \in \partial_{C} B\left(u_{t_{n}}\right), u_{t_{n}} \rightarrow \phi\left(t_{o}\right)$ and $\partial_{C} B$ is upper semicontinuous, we conclude that $w_{o} \in$ $\partial_{C} B\left(\phi\left(t_{o}\right)\right)$. On the other hand, we shall show that $v_{o} \in F\left(\phi\left(t_{o}\right)\right) \cap T_{C}\left(\phi\left(t_{o}\right)\right)$. Indeed, for $v_{n}:=\frac{\phi\left(t_{n}\right)-\phi\left(t_{o}\right)}{t_{n}-t_{o}}$,

$$
\phi\left(t_{n}\right)=\phi\left(t_{o}\right)+v_{n}\left(t_{n}-t_{o}\right) \in C \quad \text { with } \quad t_{n} \rightarrow t_{o} \quad \text { and } \quad v_{n} \rightarrow v_{o} .
$$

Hence, using (5.2), we conclude that $v_{o} \in T_{C}\left(\phi\left(t_{o}\right)\right)$. Now, to show that $v_{o} \in F\left(\phi\left(t_{o}\right)\right)$, we use (5.21) to conclude that we can always find $\alpha_{n} \in\left(t_{o}, t_{n}\right)$ such that $v_{n} \in F\left(\phi\left(\alpha_{n}\right)\right)$. Finally, since $F$ is upper semicontinuous and $\alpha_{n} \rightarrow$ $t_{o}$, we conclude that $v_{o} \in F\left(\phi\left(t_{o}\right)\right)$. Finally, if we reconsider (5.19), after passing to an adequate subsequence we obtain

$$
\lim _{n \rightarrow \infty}\left\langle w_{t_{n}}, \frac{\phi\left(t_{n}\right)-\phi\left(t_{o}\right)}{t_{n}-t_{o}}\right\rangle=\left\langle w_{o}, v_{o}\right\rangle \geq \epsilon>0
$$

However, since $w_{o} \in \partial_{C} B\left(\phi\left(t_{o}\right)\right)$ and $v_{o} \in F\left(\phi\left(t_{o}\right)\right) \cap T_{C}\left(\phi\left(t_{o}\right)\right)$, (5.14) implies that $\left\langle w_{o}, v_{o}\right\rangle \leq 0$; thus, a contradiction follows.

In order to prove item 3 , we use the same exact steps as in the proof of item 1 while picking $t_{o} \in[0, T)$ such that the following properties hold simultaneously:

- $\dot{\phi}\left(t_{o}\right)$ exists,

$-\dot{\phi}\left(t_{o}\right) \in F\left(\phi\left(t_{o}\right)\right) \cap T_{C}\left(\phi\left(t_{o}\right)\right)$

- $\left\langle\eta, \dot{\phi}\left(t_{o}\right)\right\rangle=a_{t_{o}}$ for all $\eta \in \partial_{C} B\left(\phi\left(t_{o}\right)\right)$.

Indeed, we already know that each of the latter three properties holds for almost all $t \in[0, T]$; hence, we can always find $t_{o} \in[0, T)$ that satisfies these three conditions simultaneously. Next, the contradiction reasoning leads us to (5.23). Note that, in this case, we have

$$
v_{o}:=\lim _{n \rightarrow \infty} \frac{\phi\left(t_{n}\right)-\phi\left(t_{o}\right)}{t_{n}-t_{o}}=\dot{\phi}\left(t_{o}\right) \in F\left(\phi\left(t_{o}\right)\right) \cap T_{C}\left(\phi\left(t_{o}\right)\right)
$$

and, for each $\eta \in \partial_{C} B\left(\phi\left(t_{o}\right)\right)$, we have $\left\langle\eta, \dot{\phi}\left(t_{o}\right)\right\rangle=a_{t_{o}}$. Thus, using (5.15), we conclude that $\left\langle w_{o}, v_{o}\right\rangle \leq 0$; which yields to a contradiction.

In order to prove item 2, we use the proof of item 1 in Theorem 5.2 to conclude, under (M1) and the continuity of $F$, that, when the function $B$ is nonincreasing along the solutions to $\mathcal{H}_{f}$, for each $x_{o} \in \widetilde{C}$ and $v_{o} \in F\left(x_{o}\right) \cap T_{C}\left(x_{o}\right),\left[\begin{array}{ll}v_{o} & 0\end{array}\right]^{\top} \in T_{\operatorname{epi} B \cap(C \times \mathbb{R})}\left(x_{o}, B\left(x_{o}\right)\right)$. Hence, $\left[\begin{array}{ll}v_{o} & 0\end{array}\right]^{\top} \in T_{\operatorname{epi} B}\left(x_{o}, B\left(x_{o}\right)\right)$. Next, since $B$ is Lipschitz and regular, we use Lemma A.6 to conclude (5.14).

Remark 5.21. The first item in Theorem 5.20 can be found in [9] for the unconstrained case and in [27] for the constrained case. However, the original proof proposed in this paper illustrates the reason why we cannot obtain a similar result when $B$ is discontinuous using the $\partial_{P} B$.

Example 5.22. Consider the constrained system $\mathcal{H}_{f}=(C, F)$ introduced in Example 4.3. We already showed that Assumption 2.5 holds, $F$ is locally Lipschitz, and (M1) holds. Hence, using Theorem 5.20, we conclude that a locally Lipschitz and regular function $B: \mathbb{R}^{2} \rightarrow \mathbb{R}$ is nonincreasing along the solutions to $\mathcal{H}_{f}$ if and only if (5.14) holds.

As in Theorem 5.12, (M1) can be relaxed provided that Assumptions 5.10 and 5.11 hold. 
Theorem 5.23. Consider a system $\mathcal{H}_{f}=(C, F)$ such that Assumption 2.5 holds. Let $B: \mathbb{R}^{n} \rightarrow \mathbb{R}$ be a locally Lipschitz function. Assume further that Assumptions 5.11 and 5.10 hold with $x \mapsto N_{\mathrm{epi} B \cap(C \times \mathbb{R})}^{P}(x, B(x))$ therein replaced by $x \mapsto\left[\partial_{C} B(x)^{\top}-1\right]^{\top}$ and $B$ is regular. Then,

1. When $F$ is continuous, $(\star) \Rightarrow(5.14)$.

Proof. T establish the proof, we distinguish the following two situations:

- When $(x, B(x)) \in \partial(\operatorname{epi} B) \cap(\operatorname{int}(C) \times \mathbb{R})$, according to the proof of 2. in Theorem 5.12, we notice that $(\mathrm{M} 1)$ holds trivially. Furthermore, using the proof of the necessary part in Theorem 5.2, we conclude that $\left[\begin{array}{ll}v_{o} & 0\end{array}\right]^{\top} \in T_{\text {epi } B}(x, B(x))$ for each $v_{o} \in F(x)$. Hence, (5.14) follows using Lemma A.6 since $B$ is locally Lipschitz and regular.

- Next, when $(x, B(x)) \in \partial(\operatorname{epi} B) \cap((\widetilde{C} \backslash \operatorname{int}(C)) \times \mathbb{R}),(5.14)$ follows using the same contradiction argument used in the proof of Theorem 5.12 and the fact that $N_{\mathrm{epi} B \cap(C \times \mathbb{R})}^{P}=\left[\begin{array}{ll}\partial_{C} B & -1\end{array}\right]^{\top}$, see Lemma A.6 in the appendix.

\subsection{When $B$ is locally Lipschitz and $\operatorname{cl}(C)$ is pre-contractive}

As in Corollary 5.16, when the solutions to $\mathcal{H}_{f}$ do not flow in $\partial C$ (i.e., Asm. 5.14 holds), we will show that we can use infinitesimal inequalities that we check only on the interior of the set $C$. That is, we introduce the following conditions:

$$
\begin{aligned}
& \langle\eta, \zeta\rangle \leq 0 \quad \forall \eta \in \partial_{C} B(x), \quad \forall \zeta \in F(x), \quad \forall x \in \operatorname{int}(C) . \\
& \langle\eta, \zeta\rangle \leq 0 \quad \forall \eta \in \partial_{C} B(x), \quad \forall \zeta \in F(x): \exists c \in \mathbb{R}:\langle\eta, \zeta\rangle=c \text { for all } \eta \in \partial_{C} B(x), \quad \forall x \in \operatorname{int}(C) . \\
& \langle\nabla B(x), \zeta\rangle \leq 0 \quad \forall \zeta \in F(x), \quad \forall x \in \operatorname{int}(C): \nabla B(x) \text { exists. }
\end{aligned}
$$

Corollary 5.24. Consider a system $\mathcal{H}_{f}=(C, F)$ such that Assumption 2.5 holds. Let $B: \mathbb{R}^{n} \rightarrow \mathbb{R}$ be locally Lipschitz. Then,

1. When Assumption 5.14 holds, $(5.24) \Rightarrow(\star)$.

2. When Assumption 5.14 holds and $B$ is nonpathological, $(5.25) \Rightarrow(\star)$.

3. When Assumption 5.14 holds and $F$ is continuous, (5.26) $\Rightarrow(5.24)$.

Proof. We start using Theorem 3 and the proof therein to conclude that when (5.24) holds, or (5.25) holds and $B$ is nonpathological, then $t \mapsto B(\phi(t))$ is nonincreasing along every solution $\phi: \operatorname{dom} \phi \rightarrow \operatorname{int}(C)$.

Next, using contradiction, we show that, under Assumption 5.14, if $t \mapsto B(\phi(t))$ is nonincreasing along every solution $\phi: \operatorname{dom} \phi \rightarrow \operatorname{int}(C)$ then so it is along every solution $\phi: \operatorname{dom} \phi \rightarrow \operatorname{cl}(C)$. Indeed, consider a solution $\phi: \operatorname{dom} \phi \rightarrow \operatorname{cl}(C)$ such that $t \mapsto B(\phi(t))$ fails to be nonincreasing. Using Assumption 5.14, we conclude that the solution $\phi$ cannot flow in $\partial C$. Furthermore, since $t \mapsto B(\phi(t))$ is nonincreasing in the interior $C$ and since $B$ is continuous, the map $t \mapsto B(\phi(t))$ fails to be nonincreasing under one of the two following scenarios:

- For some $\epsilon>0, B(\phi(0))<B(\phi(t))$ for all $t \in(0, \epsilon]$.

- For some $T>0$ such that $\phi(T) \in \partial C, B(\phi(T))>B(\phi(T-t))$ for all $t \in(0, \epsilon]$.

The latter two scenarios contradict the continuity of the map $t \mapsto B(\phi(t))$.

Finally, the proof of item 3 can be found in Proposition 1 of [26]. 


\subsection{When $B$ is continuously differentiable and $\tilde{C}$ is generic}

When a function $B: \mathbb{R}^{n} \rightarrow \mathbb{R}$ is continuously differentiable, $\partial_{C} B \equiv \nabla B$; hence, (5.14) becomes

$$
\langle\nabla B(x), \eta\rangle \leq 0 \quad \forall \eta \in F(x) \cap T_{C}(x), \quad \forall x \in \widetilde{C} .
$$

Similarly, (5.24) becomes

$$
\langle\nabla B(x), \eta\rangle \leq 0 \quad \forall \eta \in F(x), \quad \forall x \in \operatorname{int}(C) .
$$

The following corollaries are in order.

Corollary 5.25. Consider a system $\mathcal{H}_{f}=(C, F)$ such that Assumption 2.5 holds. Let $B: \mathbb{R}^{n} \rightarrow \mathbb{R}$ be a continuously differentiable function. Then,

1. $(5.27) \Rightarrow(\star)$.

2. When (M1) holds and $F$ is continuous, $(\star) \Leftrightarrow(5.27)$.

Proof. Using Theorem 5.20, the statement follows under (P4) and the fact that each continuously differentiable function is both locally Lipschitz and regular.

Next, using the continuity argument in Theorem 5.12 under Assumption 5.11, we will show that (M1) is also not required.

Corollary 5.26. Consider a system $\mathcal{H}_{f}=(C, F)$ such that Assumption 2.5 holds. Let $B: \mathbb{R}^{n} \rightarrow \mathbb{R}$ be a continuously differentiable function. Assume further that Assumption 5.11 holds. Then,

1. $(5.27) \Rightarrow(\star)$.

2. When $F$ is continuous, $(\star) \Rightarrow(5.27)$.

Proof. The proof follows from Theorem 5.23 while using (P4), the fact that each continuously differentiable function is locally Lipschitz and regular, and $\nabla B$ continuous.

Example 5.27. Consider the constrained system $\mathcal{H}_{f}=(C, F)$ introduced in Example 4.3. We already showed that Assumption 2.5 holds and $F$ is locally Lipschitz. Moreover, we will show that Assumption 5.11 is also satisfied. Indeed, for each $x_{o} \in \partial C \cap C$, i.e. $x_{o}=\left[\begin{array}{ll}x_{o 1} & 0\end{array}\right]^{\top}$ for some $x_{o 1} \in \mathbb{R}$, there exists $\epsilon>0$ such that $x_{\epsilon}=$ $\left[\begin{array}{ll}x_{o 1} & \epsilon\end{array}\right]^{\top} \in \operatorname{int}(C)$ can be made arbitrary close to $x_{o}$; thus, Assumption 5.11 follows. Hence, using Corollary 5.26 , we conclude that a continuously differentiable function $B: \mathbb{R}^{2} \rightarrow \mathbb{R}$ satisfies $(\star)$ if and only if (5.27) is satisfied.

\subsection{When $B$ is continuously differentiable and $\operatorname{cl}(C)$ is pre-contractive}

In this case, Corollary 5.24 reduces to the following statement.

Corollary 5.28. Consider a system $\mathcal{H}_{f}=(C, F)$ such that Assumption 2.5 holds. Let $B: \mathbb{R}^{n} \rightarrow \mathbb{R}$ be continuously differentiable. Then,

1. $(\star) \Rightarrow(5.28)$.

2. When Assumption 5.14 holds and $F$ is continuous, $(5.28) \Leftrightarrow(\star)$.

Proof. The proof follows from a direct application of Corollary 5.24 while using the fact that each continuously differentiable function is locally Lipschitz and regular, and, $\nabla B \equiv \partial_{C} B$. 


\section{Conclusion}

This paper characterizes the nonincrease of scalar functions along solutions to differential inclusions defined on a constrained set. Such a problem is shown to arise naturally when analyzing stability and safety in constrained systems using Lyapunov-like techniques. Different classes of scalar functions are considered in this paper including lower semicontinuous, locally Lipschitz and regular, and continuously differentiable functions. As a future work, one could consider replacing Assumptions (M1) and (M2) by tighter assumptions or analyze their necessity.

\section{Appendix A. Supporting RESUlts}

In this section, we recall a useful intermediate result as well as some useful properties of $\partial_{C} B$ and $\partial_{P} B[4,13]$. The following result can be found in Problem 11.23, page 67 of [13].

Lemma A.1. Let $B: \mathbb{R}^{n} \rightarrow \mathbb{R}$ be lower semicontinuous and let $(\zeta, 0) \in N_{\mathrm{epi} B}^{P}(x, B(x))$. Then, for each $\epsilon>0$, there exists $x^{\prime} \in x+\epsilon \mathbb{B}$ and $\left(\zeta^{\prime},-\lambda\right) \in N_{\mathrm{epi} B}^{P}\left(x^{\prime}, B\left(x^{\prime}\right)\right)$ such that

$$
\lambda>0, \quad\left|B\left(x^{\prime}\right)-B(x)\right|<\epsilon, \quad\left|(\zeta, 0)-\left(\zeta^{\prime},-\lambda\right)\right| \leq \epsilon .
$$

Remark A.2. According to Definition 3.5, $\partial_{P} B(x)$ is empty whenever $N_{\mathrm{epi} B}^{P}(x, B(x)) \subset \mathbb{R}^{n} \times\{0\}$. However, the set of points where $\partial_{P} B(x)$ is nonempty is dense in $\mathbb{R}^{n}$ and Lemma A.1 is a consequence of the density theorem in Theorem 3.1, page 39 of [13].

Next, we recall from Theorem 2.4, page 75 of [13] the following version of the mean-value theorem in the case of locally Lipschitz functions, which will play a fundamental role to solve Problem 3.1 when $B$ is locally Lipschitz and regular.

Lemma A.3 (Lebourg's mean value theorem). Let $(x, y) \in \mathbb{R}^{n} \times \mathbb{R}^{n}$, and suppose that $B: \mathbb{R}^{n} \rightarrow \mathbb{R}$ is locally Lipschitz. Then, there exists a point $u$ in the open line-segment relating $x$ to $y$ denoted $(x, y)$ such that

$$
B(x)-B(y) \in\left\{\langle z, x-y\rangle: z \in \partial_{C} B(u)\right\} .
$$

Remark A.4. When the function $B$ is only lower semicontinuous, since $\partial_{P} B$ is not guaranteed to exist everywhere in $\mathbb{R}^{n}$, it is not possible to formulate a mean-value theorem similar to (A.1) using $\partial_{P} B$ instead of $\partial_{C} B$ with $u$ belonging to the open segment $(x, y)$; see [10].

The following useful properties of the Clarke generalized gradient can be found in Proposition 1.5, page 73 of [13], Proposition 3.1, page 78 of [13], and Theorem 5.7, page 87 of [13]. In the following lemma, we recall only those that are useful to prove our results.

Lemma A.5. Consider a locally Lipschitz function $B: \mathbb{R}^{n} \rightarrow \mathbb{R}$. Then,

(P1) the set-valued map $\partial_{C} B$ is locally bounded and upper semicontinuous,

(P2) $\partial_{C} B(x) \neq \emptyset \quad \forall x \in \mathbb{R}^{n}$,

(P3) for each $x \in \mathbb{R}^{n}, \zeta \in \partial_{C} B(x) \Leftrightarrow$ $\left\langle\left[\zeta^{\top}-1\right]^{\top}, v\right\rangle \leq 0 \quad \forall v \in N_{\text {epi } B}(x, B(x))$,

(P4) $B$ is continuously differentiable $\Longrightarrow \partial_{C} B(x)=\{\nabla B(x)\} \quad \forall x \in \mathbb{R}^{n}$.

the following lemma is a direct consequence of Definition 3.4 and (P3).

Lemma A.6. Consider a locally Lipschitz and regular function $B: \mathbb{R}^{n} \rightarrow \mathbb{R}$. Then, for each $x \in \mathbb{R}^{n}$, (P5) $\eta \in \partial_{C} B(x) \Leftrightarrow\left[\eta^{\top}-1\right]^{\top} \in N_{\mathrm{epi} B}(x, B(x))$.

Lemma A.7. Given a subset $S \subset \mathbb{R}^{n}$, the proximal normal cone $N_{S}^{P}$ is a subset of the normal cone $N_{S}$. 
Proof. By definition, $z \in N_{S}^{P}(x)$ implies the existence of $r>0$ such that $|x+r z|_{S}=r|z|$. Let $y:=x+r z$ and note that $|y|_{S}=r|z|=|r z|=|y-x|$. Hence, $x$ belongs to the projection of $y$ on $S$. Now, using Proposition 3.2 .3 of [2], we conclude that $(y-x)=r z \in N_{S}(x)$. Finally, since $N_{K}$ is a cone and $r>0$, it follows that $z \in N_{S}(x)$.

\section{REFERENCES}

[1] A.D. Ames, X. Xu, J.W. Grizzle and P. Tabuada, Control Barrier Function Based Quadratic Programs with Application to Automotive Safety Systems (2018).

[2] J.P. Aubin, Viability Theory. Birkhauser Boston Inc., Cambridge, MA, USA (1991).

[3] J.P. Aubin and A. Cellina, Vol. 264 of Differential Inclusions: Set-Valued Maps and Viability Theory. Springer Science \& Business Media (2012).

[4] J.P. Aubin and H. Frankowska, Set-valued Analysis. Springer Science \& Business Media (2009).

[5] A. Bacciotti and F. Ceragioli, Stability and stabilization of discontinuous systems and nonsmooth Lyapunov functions. ESAIM: COCV 4 (1999) 361-376.

[6] A. Bacciotti and F. Ceragioli, Nonsmooth Lyapunov functions and discontinuous Carathéodory systems. IFAC Proc. 37 (2004) $841-845$.

[7] R.P. Boas and H.P. Boas, vol. 13 of A Primer of Real Functions, 4th edn., Mathematical Association of America (1996).

[8] F. Clarke, Vol. 264 of Functional analysis, calculus of variations and optimal control. Springer Science \& Business Media (2013).

[9] F.H. Clarke, Vol. 5 of Optimization and Nonsmooth Analysis (1990).

[10] F.H. Clarke and Y.S. Ledyaev, Mean value inequalities. Proc. Am. Math. Soc. 122 (1994) 1075-1083.

[11] F.H. Clarke, Y.S. Ledyaev, E.D. Sontag and A.I. Subbotin, Asymptotic controllability implies feedback stabilization. IEEE Trans. Autom. Control 42 (1997) 1394-1407.

[12] F.H. Clarke, Y.S. Ledyaev and R.J. Stern, Invariance, monotonicity, and applications, in Nonlinear analysis, differential equations and control. Springer (1999) 207-305.

[13] F.H. Clarke, Y.S. Ledyaev, R.J. Stern and P.R. Wolenski, Nonsmooth Analysis and Control Theory, vol. 178, Springer Science \& Business Media (2008).

[14] F.H. Clarke, R.J. Stern and P.R. Wolenski, Subgradient criteria for monotonicity, the Lipschitz condition, and convexity. Can. J. Math. 45 (1993) 1167-1183.

[15] U. Dini, Lezioni di analisi infinitesimale, vol. 1, 2. Fratelli Nistri (1907).

[16] P. Glotfelter, J. Cortés and M. Egerstedt, Nonsmooth barrier functions with applications to multi-robot systems. IEEE Control Syst. Lett. 1 (2017) 310-315.

[17] R. Goebel, R.G. Sanfelice and A.R. Teel, Hybrid dynamical systems: modeling, stability, and robustness. Princeton University Press (2012).

[18] R. Kamalapurkar, W.E. Dixon and A.R. Teel, On reduction of differential inclusions and Lyapunov stability. ESAIM: COCV 26 (2020) 24.

[19] M. Maghenem, A. Melis and R.G. Sanfelice, Monotonicity Along Solutions to Constrained Differential Inclusions, in Proceeding of the 58th IEEE Conference on Decision and Control, Nice, France (2019).

[20] M. Maghenem and R.G. Sanfelice, Characterization of Safety and Conditional Invariance for Nonlinear Systems, in Proceedings of the 2019 American Control Conference (ACC), IEEE (2019) 5039-5044.

[21] M. Maghenem and R.G. Sanfelice, Characterizations of Safety in Hybrid Inclusions via Barrier Functions, in Proceedings of the 22nd ACM International Conference on Hybrid Systems: Computation and Control, HSCC '19, ACM, NY, USA (2019) $109-118$.

[22] M. Maghenem and R.G. Sanfelice, Local Lipschitzness of Reachability Maps for Hybrid Systems with Applications to Safety, in Proceedings of the 23rd International Conference on Hybrid Systems: Computation and Control, HSCC '20, Association for Computing Machinery, New York, NY, USA (2020).

[23] E. Michael, Continuous selections. I. Ann. Math. (1956) 361-382.

[24] S. Prajna, A. Jadbabaie and G.J. Pappas, A framework for worst-case and stochastic safety verification using barrier certificates. IEEE Trans. Autom. Control 52 (2007) 1415-1428.

[25] R.T. Rockafellar and J.B.R. Wets, Vol. 317 of Variational Analysis. Springer Science \& Business Media (1997).

[26] M.D. Rossa, R. Goebel, A. Tanwani and L. Zaccarian, Piecewise structure of Lyapunov functions and densely checked decrease conditions for hybrid Systems. Math. Control Signals and Syst. 33 (2021) 123-149.

[27] R.G. Sanfelice, R. Goebel and A.R. Teel, Invariance principles for hybrid systems with connections to detectability and asymptotic stability. IEEE Trans. Autom. Control 52 (2007) 2282-2297.

[28] E. Sontag and H. Sussmann, Nonsmooth control-Lyapunov functions, in vol. 3 of Proceedings of the 34th IEEE Conference on Decision and Control (CDC). IEEE (1995) 2799-2805.

[29] E.D. Sontag, A Lyapunov-like characterization of asymptotic controllability. SIAM J. Control Optim. 21 (1983) $462-471$. 
[30] M. Valadier, Entraînement unilatéral, lignes de descente, fonctions lipschitziennes non pathologiques. C.R. Acad. Sci. Paris Sér. I Math 8 (1989) 241-244.

\section{Subscribe to Open (S2O)}

\section{A fair and sustainable open access model}

This journal is currently published in open access under a Subscribe-to-Open model (S2O). S2O is a transformative model that aims to move subscription journals to open access. Open access is the free, immediate, online availability of research articles combined with the rights to use these articles fully in the digital environment. We are thankful to our subscribers and sponsors for making it possible to publish this journal in open access, free of charge for authors.

Please help to maintain this journal in open access!

Check that your library subscribes to the journal, or make a personal donation to the S2O programme, by contacting subscribers@edpsciences.org

More information, including a list of sponsors and a financial transparency report, available at: https://www.edpsciences.org/en/maths-s2o-programme 